\title{
ARTICLE
}

Received 26 Oct 2016 | Accepted 3 Mar 2017 | Published 3 May 2017

DOl: $10.1038 /$ ncomms15151

OPEN

\section{The careg element reveals a common regulation of regeneration in the zebrafish myocardium and fin}

\author{
Catherine Pfefferli ${ }^{1} \&$ Anna Jaźwińska ${ }^{1}$
}

The existence of common mechanisms regulating organ regeneration is an intriguing concept. Here we report on a regulatory element that is transiently activated during heart and fin regeneration in zebrafish. This element contains a ctgfa upstream sequence, called careg, which is induced by TGF $\beta /$ Activin- $\beta$ signalling in the peri-injury zone of the myocardium and the fin mesenchyme. In addition, this reporter demarcates a primordial cardiac layer and intraray osteoblasts. Using genetic fate mapping, we show the regenerative competence of careg-expressing cells. The analysis of the heart reveals that the primordial cardiac layer is incompletely restored after cryoinjury, whereas trabecular and cortical cardiomyocytes contribute to myocardial regrowth. In regenerating fins, the activated mesenchyme of the stump gives rise to the blastema. Our findings provide evidence of a common regenerative programme in cardiomyocytes and mesenchyme that opens the possibility to further explore conserved mechanisms of the cellular plasticity in diverse vertebrate organs.

\footnotetext{
${ }^{1}$ Department of Biology, University of Fribourg, Chemin du Musée 10, 1700 Fribourg, Switzerland. Correspondence and requests for materials should be addressed to A.J. (email: anna.jazwinska@unifr.ch).
} 
A dult zebrafish, axolotls and newts are valuable models in regenerative biology. These animals can survive substantial heart and limb injuries, and efficiently replace the lost parts with new functional tissues ${ }^{1-10}$. During reconstruction of the missing part, remnant injury-associated cells reactivate morphogenetic programs, proliferate and recreate a replicate of the original structure. Clonal analysis and genetic lineage tracing in salamanders and zebrafish have provided evidence that specialized cell types, such as muscle cells, cardiomyocytes and osteoblasts, undergo reversion to a less mature state, referred to as dedifferentiation, within the same lineage hierarchy ${ }^{11-18}$. Lesscomplex cells, such as epithelial, mesothelial and fibroblast-like cells, can more rapidly enter the regenerative programme, and additionally, may exhibit the plasticity to undergo transition to other developmentally related tissues or even more distant lineages through transdifferentiation ${ }^{19}$. The key regenerationparticipating cells of the heart and the appendage, namely cardiomyocytes (CMs) and mesenchymal cells (MES), substantially differ in their architectural complexity, size and metabolism. An intriguing question is whether such diverse cell types activate common genetic regulatory mechanisms during the regenerative response.

Transgenic approaches in the zebrafish heart and fin showed that the regenerated tissues derive from local functional cells that can undergo limited dedifferentiation ${ }^{12-15,17,18,20}$. In the resected ventricle, analysis of the gata4 transgenic reporter revealed that cortical CMs in the vicinity of the wound reactivate developmental gene expression and contribute to the new myocardium ${ }^{13,21}$. In the amputated fin, blastema formation requires the activation of MES in the stump. The distal part of the regenerative outgrowth acts as an apical signalling centre, whereas the proximal blastema comprises the proliferation and re-differentiation zone ${ }^{22-26}$. Chromatin-remodelling factors are required for both organ regeneration ${ }^{27,28}$. The lineage tracing and genetic clonal analyses have provided no evidence for a contribution of remote stem cells to the heart and fin regenerate ${ }^{12,13,20,29}$.

Despite the anatomical differences between the heart and fin, a concept of common regulatory mechanisms of regeneration has been recently proposed. This idea is based on the identification of a short DNA sequence upstream to the zebrafish metabolic gene, leptin $b$, which is induced in the endocardium and wound tissue of the heart, as well as in the blastema of the $\mathrm{fin}^{30}$. No activation of this enhancer element, however, has been observed in CMs. Thus, it still remains unknown whether common regulatory elements exist for injury-responsive cardiac myocytes and fin fibroblasts.

The ventricle of the adult zebrafish heart is a complex structure, containing an outer cortical (compact) layer and inner trabecular (spongy) fascicles ${ }^{31}$. Both types of myocardium are separated by flattened cardiac cells, called junctional (transitional) $\mathrm{CMs}^{32,33}$. On the basis of multicolour clonal analysis, this cardiac layer has been named 'primordial' layer, which is established in the early embryonic heart and maintained throughout ontogenesis ${ }^{34}$. While the gata4-reporter studies have provided evidence for a regenerative contribution of the cortical myocardium ${ }^{13}$, the restorative potential of trabecular and primordial $\mathrm{CMs}$ remains unclear.

In this study, we identified a $3.18 \mathrm{~kb}$ DNA fragment containing an upstream genomic sequence of the ctgfa gene, called the careg element, which is activated in the regenerating ventricular CMs and fin MES in a TGF $\beta /$ Activin- $\beta$ signalling-dependent manner. Our findings yield insight into the mechanisms of heart and fin restoration and provide evidence for the existence of regeneration regulatory programs that are conserved between architecturally diverse cells, such as cardiomyocytes and mesenchymal cells.

\section{Results}

A reporter of peri-injured cardiomyocytes and fin mesenchyme. To uncover the mechanisms underlying the injury-dependent reactivation of morphogenetic programs in distinct organs, we searched for an informative DNA regulatory element that is induced in regenerating myocardium and fin. In both organs, injury-activated tissues upregulate synthesis of specific developmental proteins. In the heart, a portion of the trabecular myocardium within $100 \mu \mathrm{m}$ from the injury border reactivates the expression of the embryo-specific cardiac myosin heavy chain isoform, embCMHC ${ }^{35}$. In the fin, MES reorganization occurs within at least $300 \mu \mathrm{m}$ from the amputation plane, which is associated with the upregulation of Tenascin C, a de-adhesive extracellular matrix protein ${ }^{36}$. Using a candidate approach, we identified a transgenic reporter that overlaps with the transient expression of embCMHC and Tenascin $\mathrm{C}$ in the regenerating hearts and fins, respectively. The reporter was driven by a $3.18 \mathrm{~kb}$ upstream regulatory sequence of connective tissue growth factor a $(\operatorname{ctg} f a)^{37}$. This transgenic line was originally established to investigate notochord development, and was also used to highlight the connective tissue of regenerating fins ${ }^{37,38}$, but it had not yet been investigated in the heart. Thus, the activation of ctgfa:EGFP in the embCMHC-positive region was an unexpected finding.

To determine the spatiotemporal pattern of the reporter activity during organ regeneration, we analysed the dynamics of ctgfa:EGFP after ventricular cryoinjury and fin amputation. In uninjured hearts, this reporter was detected in a fine sheet of cells along the ventricular circumference (Supplementary Fig. 1a,b). At 1 d.p.ci. (days post-cryoinjury), the injured myocardium was recognized by the absence of ctgfa:EGFP expression in the outer heart layer, as opposed to the intact part of the ventricular wall (Fig. 1a,b). No ctgfa:EGFP expression was observed in the trabecular myocardium (Fig. 1c). At 4, 7 and 14 d.p.ci., fluorescence persisted in the subcortical layer, and additionally, it emerged in $\sim 60 \%$ of the cardiac muscle within a distance of $100 \mu \mathrm{m}$ from the injury border (Fig. 1d-f; Supplementary Fig. 1c-f). Approximately $20 \%$ of these cells also expressed embCMHC at 7 and 14 d.p.ci. (Fig. 1f; Supplementary Fig. 1i). Towards the end of the regenerative process at 30 d.p.ci., the expression of the reporter became reduced, and it was nearly undetectable within the new myocardium at 60 d.p.ci. (Fig. 1g,i; Supplementary Fig. 1g,h). We concluded that ctgfa:EGFP represents a reliable marker of adult CMs that transiently reactivate the regenerative programme in the peri-injured myocardium.

Consistent with previous studies of zebrafish fin regeneration $^{38}$, live-imaging of caudal fins revealed that ctgfa:EGFP was strongly upregulated in the regeneration zone at 1 and 3 d.p.a. (days post amputation), and declined when the restorative process was completed at 20 d.p.a. (Fig. 1j-o; Supplementary Fig. $2 \mathrm{a}-1)$. At 1 and 3 d.p.a., the regeneration zone included not only the outgrowing blastema, but also the peri-injury stump tissue within a distance of three ray segments below the amputation plane. Immunofluorescence analysis of longitudinal fin sections at 3 d.p.a. showed that the expression of ctgfa:EGFP was induced in the Tenascin-C-positive MES of the stump and the blastema (Fig. 1q). No expression was observed in dedifferentiated osteoblasts above the injury plane (Fig. 1s). Little fluorescence was detected in the distal blastema (Supplementary Fig. 2m,n). Taken together, ctgfa:EGFP is transiently upregulated during dedifferentiation in both CMs and MES at the injury border of the heart and the fin, respectively.

Although ctgfa:EGFP was strongly induced in the regeneration zone, we observed its homeostatic expression in the intact heart 

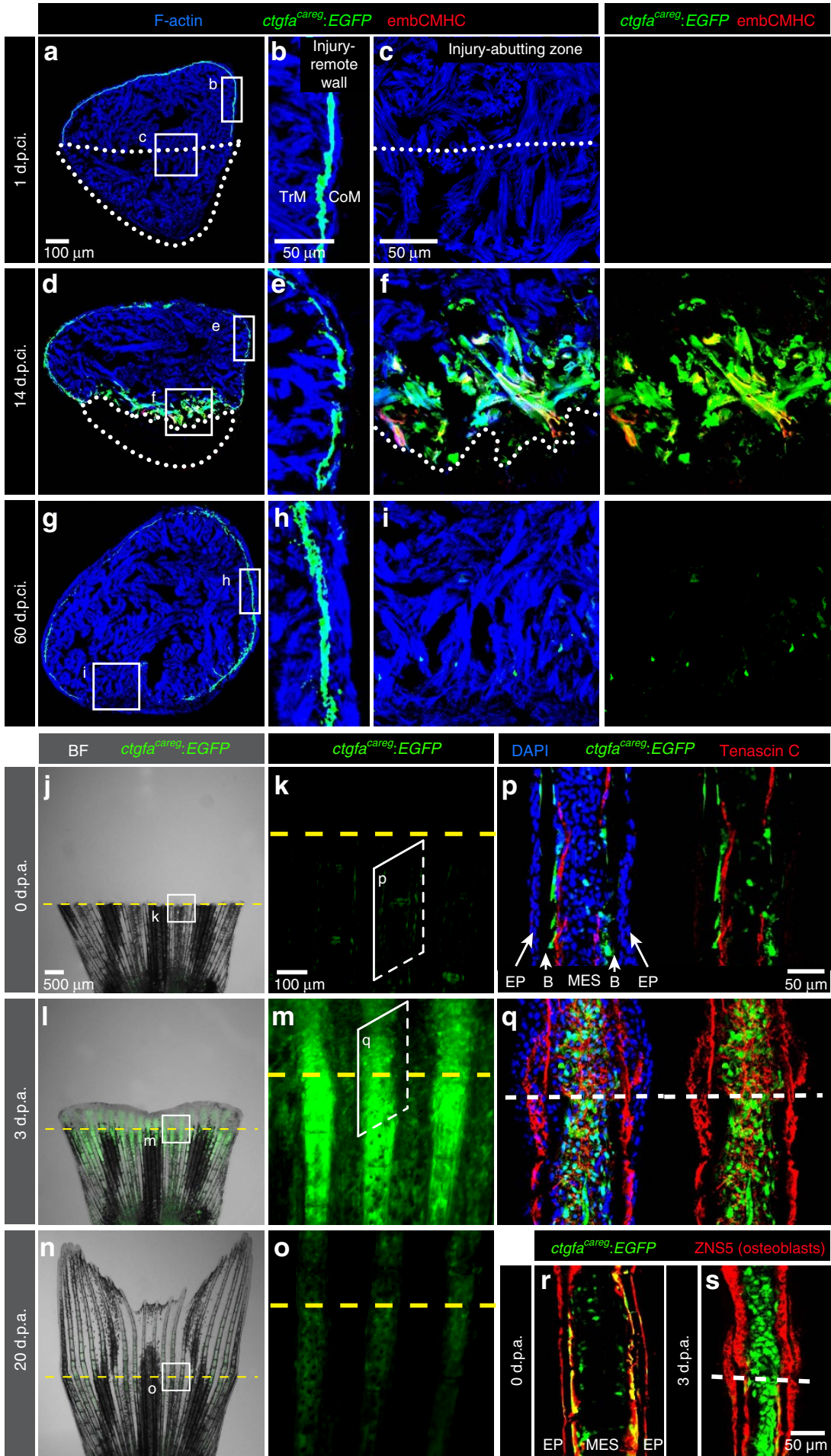

Figure 1 | A transgenic reporter of the peri-injured myocardium and the fin stump. (a-i) Transversal ventricle sections of transgenic fish ctgfacareg:EGFP immunostained for embCMHC (red) at different days post-cryoinjury (d.p.ci.). The cardiac muscle is detected by F-actin staining (Phalloidin, blue). $(\mathbf{b}, \mathbf{e}, \mathbf{h})$ The injury-remote part of the ventricular wall displays a subcortical layer of ctgfacareg:EGFP cells that is located between the thin cortical myocardium $(\mathrm{CoM})$ and the inner trabecular myocardium (TrM). This layer does not express embCMHC and remains unaltered during regeneration. (c,f,i) The injuryabutting zone of the ventricular wall shows transient expression of ctgfacareg:EGFP and embCMHC within a distance of $100 \mu \mathrm{m}$ from the wound border during regeneration. $N \geq 4$. (j-o) Live-imaging of fins at different days post amputation (d.p.a.). Bright-field (BF) was combined with fluorescence.

$(\mathbf{k}, \mathbf{m}, \mathbf{o})$ Higher magnifications of the region at the amputation plane (yellow dashed line) show transient expression of ctgfacareg:EGFP in the stump and the regenerate. $N=4$. (p-s) Immunofluorescence staining of longitudinal fin sections. (p,r) At 0 d.p.a., ctgfacareg:EGFP ${ }^{+}$cells are associated with Tenascin $C$ fibres along the border between the bones (B) and mesenchymal cells (MES), but not the epidermis (EP). Some of these cells express an osteoblast marker visualized with Zns5 antibody (red). (q,s) At 3 d.p.a., Tenascin C demarcates the zone of tissue remodelling in the stump and the outgrowth. ctgfa ${ }^{\text {careg:EGFP }}$ is upregulated in the activated MES at the peri-injury zone, but not in Zns5-labelled osteoblasts of the outgrowth. $N \geq 4$. Post-infarcted ventricle is encircled with a dotted line. Fin amputation plane is shown with a dashed line. The same rules apply to all subsequent figures. 
and fin, namely in the subcortical layer along the ventricular circumference and in cells along the interface between the bones and MES, respectively (Fig. 1b,e,h,p). In intact fins, ctgfa:EGFPexpressing cells colocalized with Zns5-labelled osteoblasts, predominantly at the interface between acellular bone matrix and MES in the intrarays (Fig. 1r). These cells were associated with extracellular de-adhesive Tenascin C, suggesting local tissue remodelling between MES and the bones (Fig. 1p,r). Thus, ctgfa:EGFP is not solely a marker of injury-abutting cells, but also of certain junctional cells located at tissue borders during homeostasis.

A distinct expression of ctgfa:EGFP and endogenous ctgfa. To determine whether the ctgfa:EGFP reporter matches the endogenous expression pattern of the ctgfa gene, we performed in situ hybridization on sections of hearts at 14 d.p.ci. and fins at 3 d.p.a. Unexpectedly, we found that anti-sense probes against ctgfa and enhanced green fluorescent protein (egfp) labelled different cells (Fig. 2a-h). ctgfa mRNA was present solely in the wound tissue of the heart, and in cells associated with the bones of fins (Fig. 2a,b,e,f). By contrast, egfp was detected in CMs along the ventricular circumference and in the vicinity of the injury of the heart, and in the blastema of the fin regenerate (Fig. 2c,d,g,h). Thus, in both organs, the egfp mRNA distribution reproduced the pattern observed by fluorescence emission, while the endogenous ctgfa gene had a distinct expression pattern. We concluded that the ctgfa:EGFP transgene contains a sequence that is regulated in a unique manner in the peri-injury zone of regenerating organs. Accordingly, we renamed this reporter, 'careg' (ctgfa reporter in regeneration), to distinguish it from the endogenous locus that includes additional genomic sequences regulating ctgfa mRNA expression. Below, we used careg to refer to this reporter.

To verify that the specificity of the careg:EGFP expression pattern is related to the $3.18 \mathrm{~kb}$ ctgfa upstream sequence and not to the genomic integration site of the construct, we created another transgenic line. We used a fluorescent protein with a rapid turnover, namely destabilized monomeric Kusabira-Orange 2 (dmKO2), which contains a C-terminal PEST domain targeted for rapid degradation via ubiquitinylation ${ }^{39}$. Double transgenic fish, careg:EGFP;careg: $d m K O 2$ displayed an overlapping pattern in regenerating CMs and MES (Fig. 2i-m). The dynamics of careg: $d m K O 2$ during heart and fin regeneration was similar to that of careg:EGFP (Supplementary Figs 3,4). On the basis of the same expression pattern of both constructs, we concluded that careg indeed contains a regulatory element that is specifically activated during regeneration.

To determine the features of the careg sequence, we performed pairwise alignment of the ctgfa genomic region between zebrafish and 9 other fish species from distinct phylogenetic orders, as well as with Xenopus, chicken, mouse and human (Fig. 3a,b). This analysis identified two conserved regions within the careg sequence, namely, a $150 \mathrm{bp}$ fragment immediately before the transcription start site and a $400 \mathrm{bp}$ region at $\sim 1 \mathrm{~kb}$ upstream from this position (Fig. 3b). The latter is conserved in five other fish species from different orders, namely cavefish, spotted gar, tilapia, platyfish and a lobe-finned fish Coelacanth (Latimeria). Interestingly, this region was also present in Xenopus and chicken, but not in mammals or in certain fish, such as medaka, fugu, stickleback or tetraodon. Bioinformatics analysis of the entire careg sequence predicted several different transcription factor-binding sites, such as Smad3, TCFF, MEF3, NKXH and GATA (Fig. 3c; Supplementary Data 1). We concluded that the careg element consists of a combination of evolutionary conserved and unique sequences that might be regulated by multiple transcription factors.
The careg reporter in various injury models. To test whether the careg reporter is activated in other injury models, we switched between the procedures used for each organ, namely, we performed amputations of the ventricular apex and cryoinjury of the caudal $\mathrm{fin}^{40,41}$. Examination of longitudinal heart sections at 7 d.p.a. revealed that careg:EGFP was induced within $100 \mu \mathrm{m}$ in the peri-injury zone of the myocardium, which also upregulated embCMHC expression (Fig. 4a,b). Thus, both methods, cryoinjury and resection, induce a local activation of careg:EGFP in CMs along the damaged border.

As opposed to the fin amputation model, the non-surgical exposure to the cold blade along the cryoinjury plane results in a progressive and spontaneous tissue detachment that is apparent between 12 and $48 \mathrm{~h}$ after procedure ${ }^{41}$. Analysis of fins shortly after cryoinjury did not reveal any expression of careg:EGFP (Fig. 4c,d). During shedding of the damaged distal part, at 1 and 2 d.p.ci., careg:EGFP was weakly induced in the stump, suggesting that the clearance of dead tissues was associated with a concomitant activation of the regenerative programme in the remaining cells (Fig. 4e,h). Remarkably, the expression of careg:EGFP became further upregulated at 4 d.p.ci., when the regenerative process was resumed after reparation of the distorted margin (Fig. 4i,j). As in amputated fins, in situ hybridization on longitudinal fin sections showed that the expression of the reporter was not associated with the induction of the endogenous ctgfa gene within the activated MES (Supplementary Fig. 5a-d). At the advanced regenerative phase, at 14 d.p.ci., careg:EGFP was downregulated, consistent with the amputation model (Fig. 4k,l).

To further test the regenerative response of the careg element, we performed a regeneration assay of the larval tail, in which the posterior tip of the notochord was truncated. Consistent with previous ctgf $a$ studies $^{37}$, careg: $d m K O 2$ displayed a basal expression level in the larval notochord (Supplementary Fig. 5e). The amputation site, however, transiently upregulated careg:dmKO2 levels during regeneration (Supplementary Fig. 5f-i). Taken together, the careg reporter is activated in regenerationresponsive cells in several injury models, suggesting its general suitability for studying organ regeneration in zebrafish.

The activation of careg is dependent on TGF $\beta$ and Activin- $\beta$. Both heart and fin regeneration requires TGF $\beta /$ Activin- $\beta$ signalling ${ }^{36,42}$. In the fibrotic tissue of the injured ventricle, this pathway also induces a transient deposition of certain matrix components, such as a remodelling protein Tenascin C, fibrilforming Collagen I and fibril-associated Collagen XII (refs 42,43). To investigate whether TGF $\beta$ /Activin- $\beta$ signalling is active in the careg expression zone of regeneration-activated CMs and MES, we analysed phosphorylation of Smad3, which is a canonical downstream signal transducer of this pathway ${ }^{44}$. Ventricle sections at 7 d.p.ci. revealed that $57.3 \% \pm 7.1 \quad(N=6)$ of careg:EGFP-positive cells contained pSmad3-labelled nuclei (Fig. 5a-c). Fin sections at 3 d.p.a. showed $70.3 \% \pm 1.8$ $(N=7)$ of careg:EGFP/pSmad3 double-positive cells in the MES compartment at $200 \mu \mathrm{m}$ below the amputation plane (Fig. 5o,p). This suggests that the peri-injury zone of both organs is substantially activated by TGF $\beta /$ Activin- $\beta$ signalling.

To test the requirement of this pathway for careg expression, we used the pharmacological inhibitor of the TGF $\beta /$ Activin- $\beta$ type I receptor, SB431542 (refs 36,42). In uninjured heart, the prolonged treatment for 7 days with this drug did not affect the homeostatic expression of careg:EGFP in the outer layer of the ventricle (Supplementary Fig. 6a-c). Consistently, at 7 and 14 d.p.ci., the inhibition of the TGF $\beta$ /Activin- $\beta$ pathway did not display any effect on reporter expression along the intact myocardial wall of careg:EGFP and careg: $d m K O 2$ hearts. By 

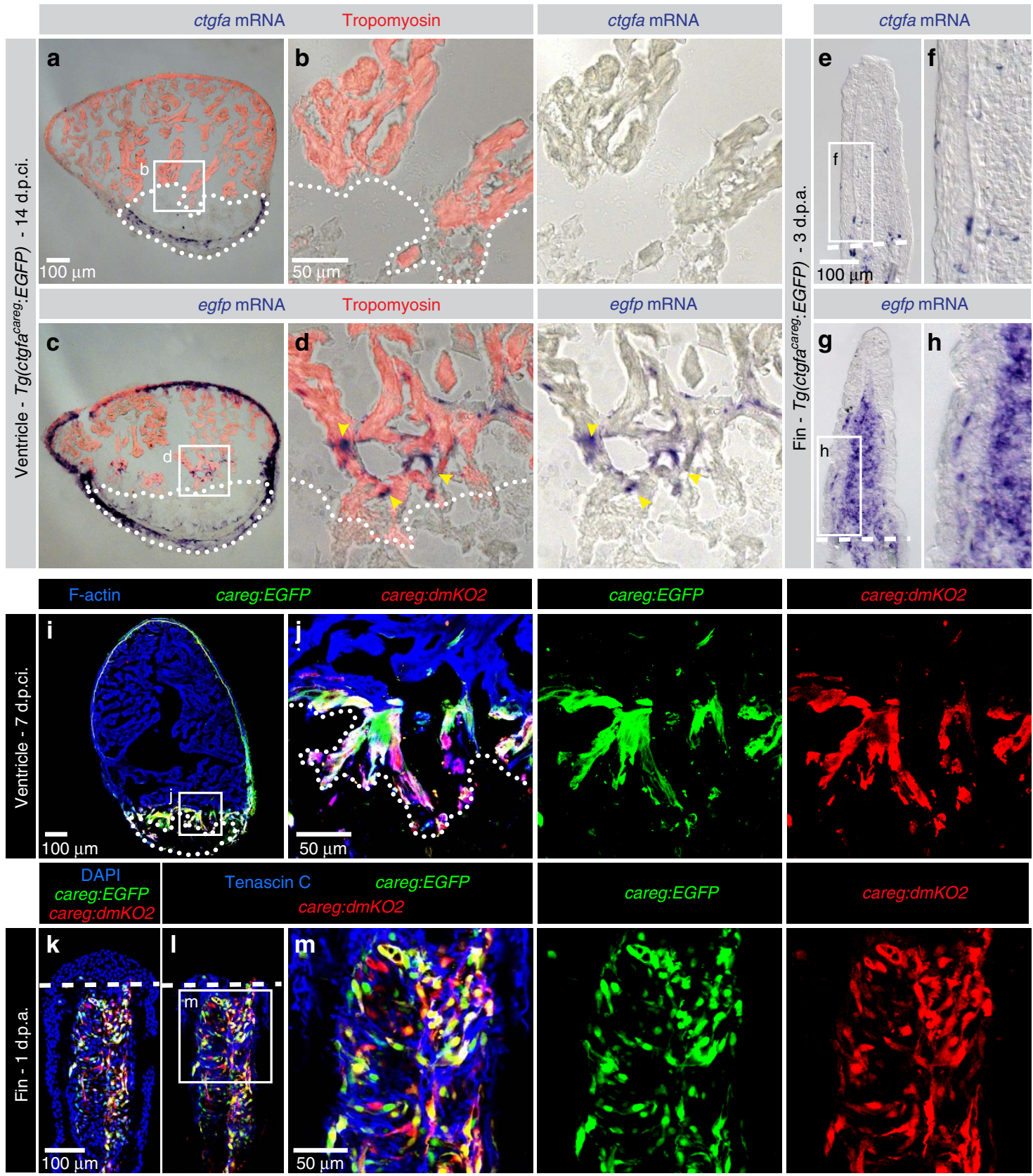

Figure 2 | ctgfa $^{\text {careg; }}$;GFP does not reproduce endogenous ctgfa expression in peri-injury tissues. (a-h) In situ hybridization using ctgfa and enhanced green fluorescent protein (egfp) probes on sections of hearts at 14 d.p.ci. and fins at 3 d.p.a. of ctgfacareg:EGFP transgenic fish shows distinct expression patterns. (a,b) ctgfa mRNA is not detected in the myocardium, but in a subset of non-myocytes in the post-infarcted tissue. (c,d) egfp mRNA is expressed in the outer wall of the myocardium and at the peri-injury zone (yellow arrowheads). (e,f) ctgfa mRNA is present in a few bone-associated cells of the fin, while $(\mathbf{g}, \mathbf{h})$ egfp mRNA is detected in the blastema. $N=6$. (i-m) careg:EGFP and careg:dmKO2 double transgenic fish display an overlapping expression pattern in the regenerating heart at 7 d.p.ci. and the fin at 1 d.p.a. $N=4$.

contrast, the peri-injury myocardium showed a three- to fourfold reduction of careg-labelled CMs (Fig. 5d-h, Supplementary Fig. 7a-j). This phenotype was also associated with attenuated embCMHC immunolabelling. We concluded that the regenerative induction of the careg element and the endogenous embCMHC expression in the peri-injured myocardium is regulated by the TGF $\beta /$ Activin $-\beta$ signalling pathway.

Restorative cardiogenesis requires CM proliferation. To determine the effect of SB431542 treatment on CM proliferation specifically in the careg-positive regenerating myocardium, we assessed BrdU incorporation within the region of $100 \mu \mathrm{m}$ from the injury border. This analysis revealed a fourfold decrease in the number of $\mathrm{Mef} 2 \mathrm{c} / \mathrm{BrdU}$ double-positive cells after inhibitor treatment (Supplementary Fig. 7k-o). Thus, the inhibition of TGF $\beta /$ Activin $-\beta$ signalling affects not only dedifferentiation, but also proliferation of CMs in the peri-injury zone.

Next, we analysed the regulation of careg:EGFP in regenerating fins. In uninjured fins, the prolonged treatment for 7 days with SB431542 did not affect the homeostatic expression of careg:EGFP in osteoblasts (Supplementary Fig. 6d,e). At 1 and 3 d.p.a., however, the inhibition of TGF $\beta /$ Activin- $\beta$ strongly prevented the regenerative outgrowth formation ${ }^{36}$, and the 
a

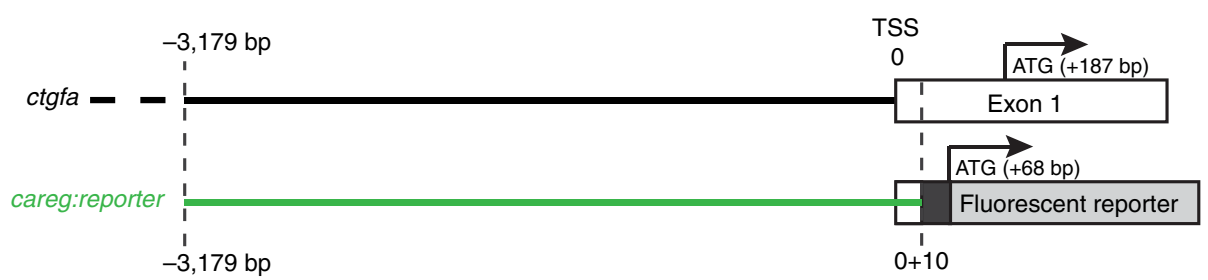

b

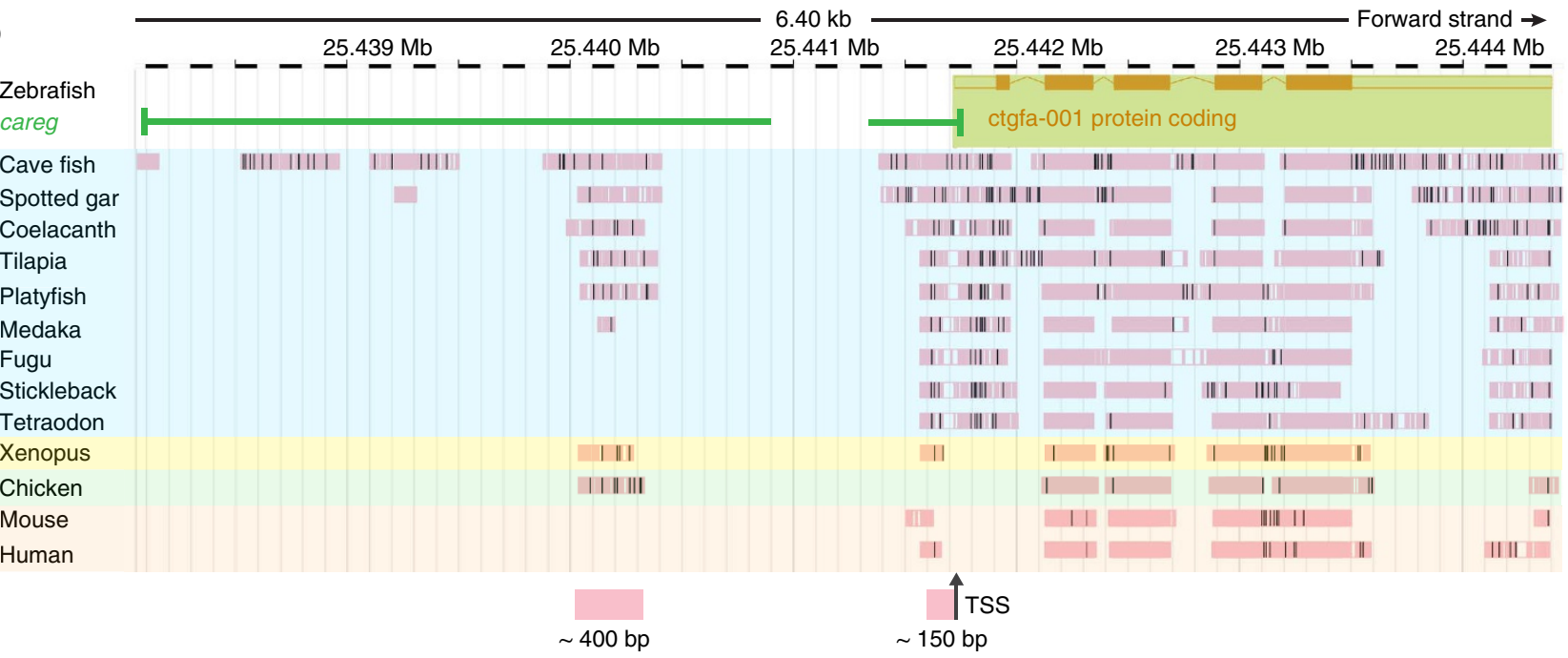

C

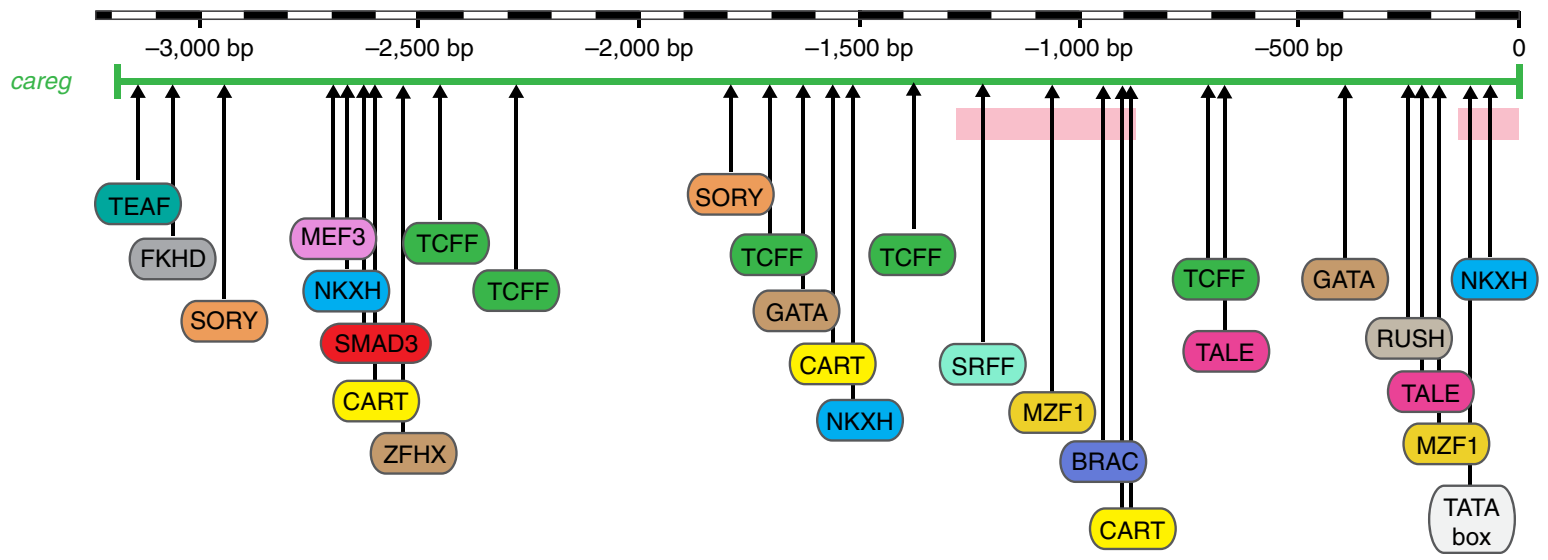

Figure 3 | Sequence analysis of the careg element. (a) Comparison of the careg element and the ctgfa genomic sequence of wild-type AB strain zebrafish, which was used to generate transgenic fish. The careg element includes a 3,179 bp sequence immediately upstream of the transcriptional start site (TSS) and $10 \mathrm{bp}$ of the $5^{\prime}$-untranslated region of ctgfa. (b) LASTz net pairwise alignment of the ctgfa genomic region between Zebrafish (Danio rerio, Ensembl

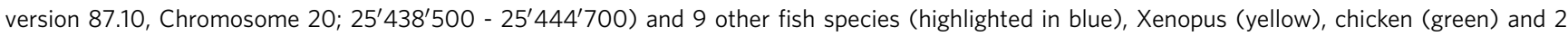
mammals (orange). The pink boxes indicate the conserved regions in the majority of the species. Black and white bars within the pink boxes represent gaps in the alignments. The careg sequence is indicated with a green line above the alignment. The gap in the careg sequence corresponds to additional 465 bp in the sequence from the Ensembl database. This sequence is absent in the genome of AB zebrafish strain. The scale bar indicates the genomic position in the chromosome 20 of zebrafish. Two conserved regions of $\sim 400$ and 150 bp are indicated with pink boxes at the bottom of the alignment. (c) Prediction of transcription factor (TF) binding sites in the careg sequence (green) with MatInspector (Genomatix). The pink boxes indicate the conserved regions identified in b. A SMAD3 binding site is highlighted in red. The complete list of TF binding sites is given in Supplementary Data 1.

activation of careg:EGFP in the stump (Fig. 5i-l; Supplementary Fig. 8a-d). To determine whether careg:EGFP expression was suppressed due to a general regenerative failure, we tested the fof $20 a$ mutant fish, called $d o b$, which fail to form a blastema ${ }^{45}$. Despite an absence of the regenerative outgrowth, dob mutant fins normally induced careg:EGFP in the peri-injured stump at 1 and 3 d.p.a. (Fig. 5m,n; Supplementary Fig. 8e-f). We concluded that the regulation of the careg reporter in the fin is dependent on TGF $\beta$ /Activin- $\beta$ signalling, irrespectively of the ability to form a blastema.
Although Fgf20a and TGF $\beta /$ Activin- $\beta$ signalling have been described as the early inducers of blastema formation, the interaction between both pathways remains unknown. Using the careg element and pSmad3 immunofluorescence, we addressed the question whether the function of TGF $\beta /$ Activin- $\beta$ signalling is dependent on Fgf20a. Our analysis of longitudinal fin sections revealed that the pSmad3 activity was blocked after SB431542 treatment, but remained unchanged in dob mutant fins (Fig. 5o-t). This finding indicates that TGF $\beta$ /Activin- $\beta$ acts independently of the Fgf20a activity during fin regeneration, providing a 

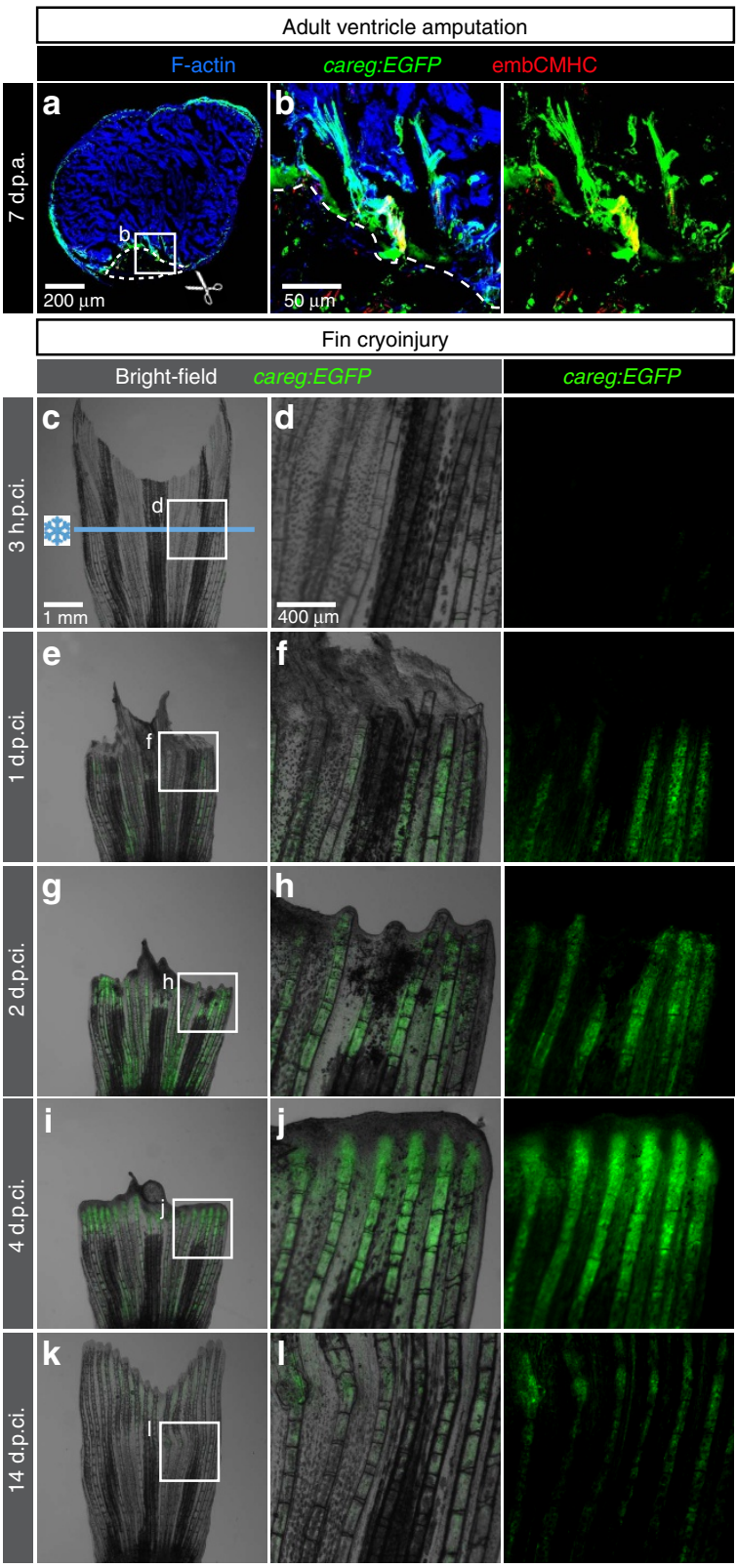

Figure 4 | The careg reporter is activated in other injury models of the zebrafish heart and fin. $(\mathbf{a}, \mathbf{b})$ Ventricle section of careg:EGFP heart at 7 days post amputation (d.p.a.) labelled with $\mathrm{F}$-actin (blue) and antibodies against GFP (green) and embCMHC (red). careg:EGFP expression is activated in the ventricular trabeculae close to the amputation plane of the resected apex (dashed line). $N=3$. (c-I) Live-imaging of the same fin at different time points post-cryoinjury. Cryoinjury of the caudal fin results in spontaneous sloughing of destroyed tissue within two days after the damage induction, followed by resumed regeneration. Bright-field was combined with fluorescence. (c,d) At 3 h.p.ci. (hours post-cryoinjury), careg:EGFP is not detected in the fin. Ischaemic tissue, which is distal to the cryoinjury plane (blue line), remains integrated with the rest of the body at this early stage. (e,f) At 1 d.p.ci., the majority of the damaged tissue detached from the stump. careg:EGFP is detected proximally to the damaged zone. (g,h) At 2 d.p.ci., the reparation of the distorted margin is accompanied by careg:EGFP expression. (i,j) At 4 d.p.ci., the protruding blastema displays enhanced expression of the careg reporter. $(\mathbf{k}, \mathbf{l})$ The advanced regeneration is associated with downregulation of careg:EGFP expression. $N=3$. new insight into the molecular network guiding blastema formation.

Taken together, the analysis of hearts and fins revealed that the same molecular pathway, TGF $\beta /$ Activin- $\beta$, stimulates the upregulation of careg in distinct regeneration-participating tissues, such as CM and MES, but not in the fibrotic tissue.

careg in primordial CMs during heart development. Heart regeneration has been proposed to involve a reactivation of cardiac embryonic programs ${ }^{3-5}$. To determine whether the induction of the careg reporter in the peri-injured CMs demarcates less differentiated CMs, we performed analysis of the embryonic heart. We found that at 1 and 3 days postfertilization (d.p.f.), careg:EGFP and careg:dmKO2 fully colocalized with embCMHC in embryonic CMs (Fig. 6a-f; Supplementary Fig. 9a,b). This finding suggests that the careg regulatory element represents a common molecular marker of activated CMs during regeneration and immature CMs during development.

To investigate the reporter expression in subsequent developmental stages, we crossed careg:EGFP with the cmlc2:DsRed-nuc line, which labels cardiac nuclei. In larvae at 12 d.p.f., we found that careg expression persisted in CMs of the heart wall, and a few careg-expressing cells were scattered in the ventricular chamber (Fig. 6g-i; Supplementary Fig. 9c). These inner CMs probably detached from the heart surface in the process of trabeculation, which involves delamination of CMs from the outer wall into the heart chamber ${ }^{46}$. Consistently, after completion of the trabeculation at 30 d.p.f., careg:EGFP and careg:dmKO2 were restricted to the outer myocardial wall (Fig. 6j-o). This result indicates that the reporter expression labels the primordial cardiac wall throughout development.

To genetically test the hypothesis that careg-positive CMs in the larval heart contribute to the delamination process of trabecular CMs, we performed lineage tracing of these cells. We generated a transgenic line with a tamoxifen-dependent Cre recombinase driven by the careg regulatory sequence, named Tg(careg:CreERT2). We crossed this strain with the ubiquitin:loxP-EGFP-STOP-loxP-mCherry lineage-tracing line, referred to as $u b i$ :Switch, which marks cells with red fluorescence after Crelox mediated recombination (Fig. 7a). To label embryonic caregpositive cells, we pulsed embryos at $75 \%$ epiboly stage with 4-hydroxytamoxifen (4-OHT) for two days and analysed the hearts at 21 d.p.f. (Fig. 7b). We found that nearly $60 \%$ of CMs $(58.2 \pm 2.5 ; \quad N=9)$ within both the trabecular and outer myocardium were randomly demarcated by mCherry expression, whereas no mCherry was observed in embryos treated with the vehicle (Fig. 7c). This fate-mapping result reveals that caregpositive embryonic CMs contribute to the trabecular myocardium during development.

careg in the primordial/junctional layer of adult hearts. Next, we examined both fluorescent transgenic reporters, careg: $d m \mathrm{KO} 2$ and careg:EGFP, in the outer wall of the adult heart. Heart sections revealed a strong co-expression of the fluorescent proteins in a single subcortical cell layer between the compact and trabecular myocardium (Fig. 8a-c). This cell layer co-expressed cmlc2:EGFP and Tropomyosin, confirming the cardiac identity. Considering developmental expression of careg and the recent multicolour clonal analysis ${ }^{34}$, we concluded that the careg reporter is a marker of the 'primordial' CMs in the zebrafish ventricle.

To determine the role of the primordial layer in the adult heart, we analysed features of the careg-expressing CMs. First, Tropomyosin revealed a reduced pattern of sarcomeres in 

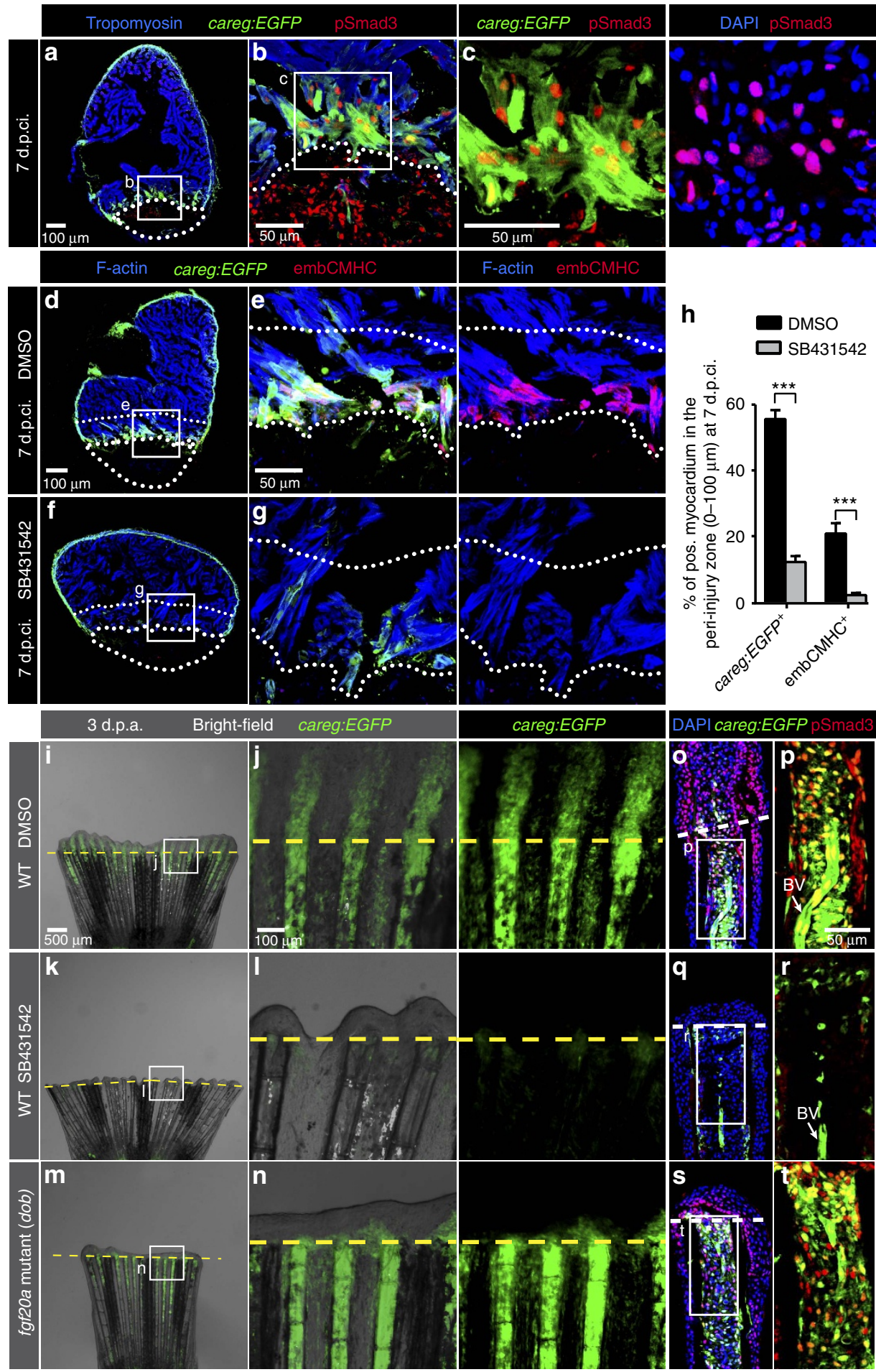

Figure 5 | The regeneration biosensor careg is dependent on TGF $\boldsymbol{\beta} /$ Activin- $\boldsymbol{\beta}$ signalling. (a-c) Immunofluorescence staining of careg:EGFP ventricle at 7 d.p.ci. with antibodies against GFP (green), pSmad3 (red) and Tropomyosin (blue) revealed the presence of TGF $\beta /$ Activin- $\beta$-activated cells in the careg:EGFP-expressing tissue. $N=6$. ( $\mathbf{d}-\mathbf{g})$ careg:EGFP heart sections at 7 d.p.ci. treated with $0.1 \%$ DMSO or $20 \mu M$ SB431542, an inhibitor of TGF $\beta$ type I receptors, and immunostained with antibodies against GFP (green) and embCMHC (red). The intact myocardium was detected with F-actin staining (blue). In the magnified images, the upper dotted line demarcates a $100 \mu \mathrm{m}$-thick margin of the remaining myocardium from the injury border (lower dotted line). (h) Percentage of careg:EGFP ${ }^{+}$and embCMHC ${ }^{+}$area within a distance of $100 \mu \mathrm{m}$ from the post-infarcted tissue in hearts at 7 d.p.ci. treated with DMSO or SB431542. The inhibitor treatment resulted in a significant reduction of careg:EGFP and embCMHC expression in the peri-injured zone, compared to control hearts treated with DMSO. $N=8 .{ }^{\star \star \star} P<0.001$; unpaired t-test. Error bars correspond to s.e. of the mean (s.e.m.). (i-n) Live-imaging of careg:EGFP fins at 3 d.p.a. in a wild-type (WT) background treated with DMSO or SB431542, and in the fgf20a (dob) mutant background. careg:EGFP expression is suppressed in the stump by TGF $\beta$ inhibition, but it remains normal in fgf2Oa mutant fins. (o-t) Longitudinal sections of the fins shown in the left panels display a reduction of pSmad3-positive nuclei (red) and careg:EGFP in the stump of SB431542-treated fins, whereas both markers are unaltered in fgf20a mutants. BV, blood vessel. $N=4$. 

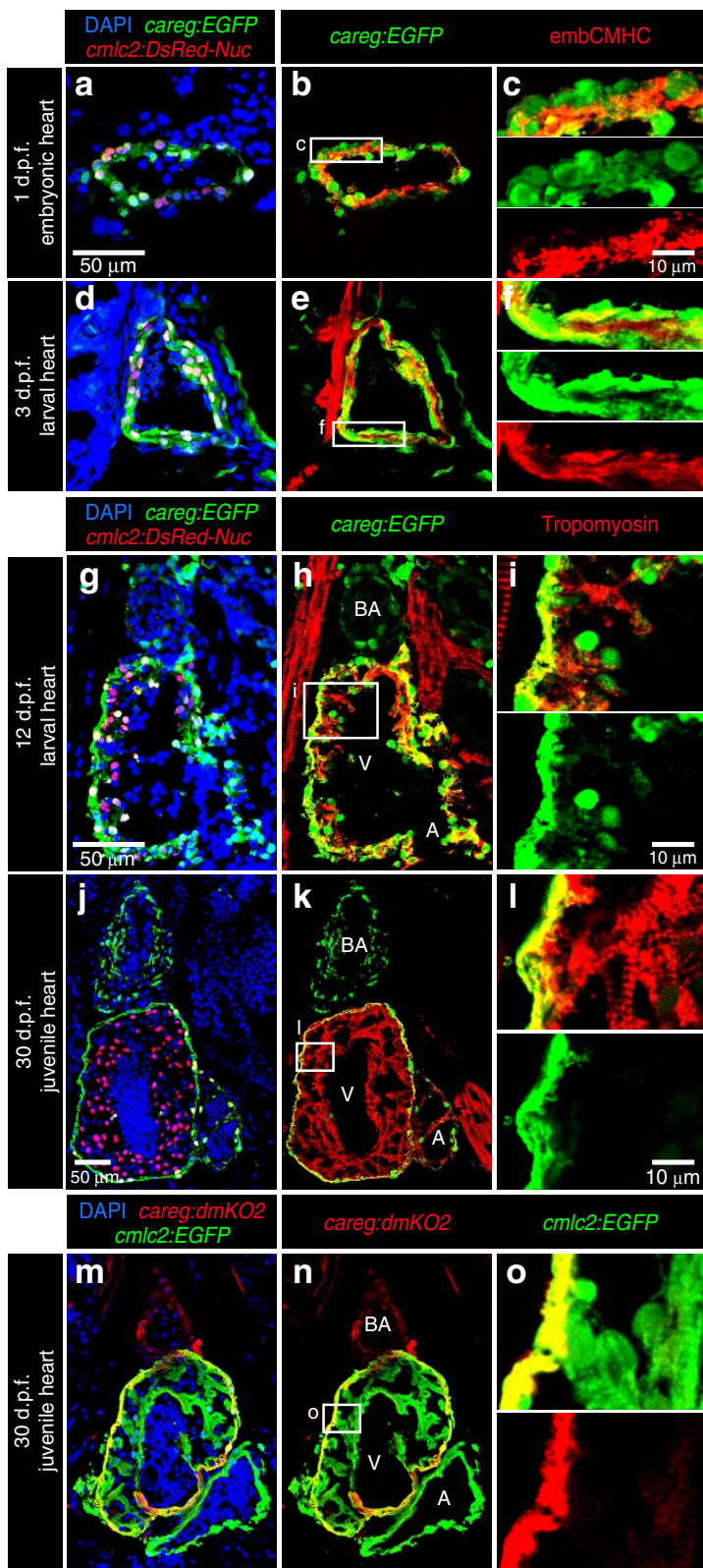

Figure 6 | careg is expressed in embryonic CMs and the outer wall of developing ventricle. (a-I) Longitudinal sections of careg:EGFP;cmlc2:DsRed2Nuc double transgenic hearts at different time points during development. Cardiac nuclei are marked by DsRed expression. The endogenous fluorescence was quenched with $\mathrm{HCl}$ treatment before immunostaining. GFP and DsRed were detected by antibody staining. (a-f) At 1 and 3 d.p.f. (days post-fertilization), careg:EGFP and endogenous embCMHC are coexpressed in embryonic CMs. (g-i) At 12 d.p.f., a few careg:EGFP ${ }^{+}$CMs delaminate from the outer heart surface and invade into the ventricle (V) chamber, as seen by the residual EGFP. (j-I) At 30 d.p.f., careg:EGFP is restricted to the outer layer of the ventricular wall, and is downregulated in the trabecular myocardium. careg:EGFP is also expressed in non-myocytes of the bulbus arteriosus (BA) and a few cells of the atrium (A). (m-0)

Longitudinal sections of careg:dmKO2; cm/c2:EGFP transgenic heart at 30 d.p.f. immunostained for GFP (green) and dmKO2 (red). The careg:dmKO2 transgenic line has a cardiac developmental expression pattern similar to that of careg:EGFP (Supplementary Fig. 10). $N \geq 5$.

careg-expressing cells, especially in comparison to that of cortical CMs, which were typically rod-shaped with a very distinct striation (Fig. 8b). This suggests that careg-positive CMs possess a
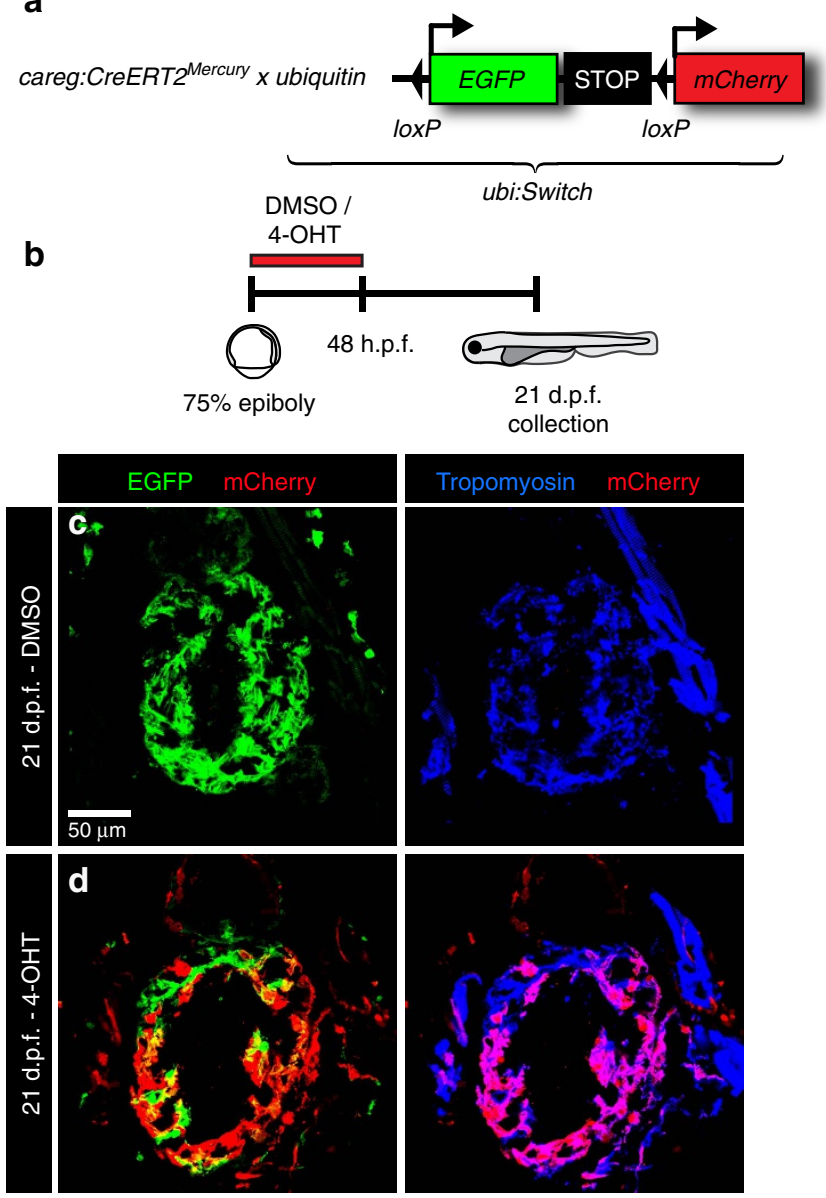

Figure 7 | Embryonic careg-positive CMs contribute to the trabecular myocardium. (a) Schematic representation of the transgenic strains used for lineage tracing. (b) Experimental design. (c,d) Longitudinal sections of hearts at 21 d.p.f. immunostained against mCherry (red), GFP (green) and Tropomyosin (blue). (c) Control embryos treated with the vehicle do not display mCherry fluorescence. (d) mCherry ${ }^{+} \mathrm{CMs}$ are present in the trabecular and outer myocardium at 21 d.p.f. in embryos treated with 4-OHT, suggesting that the trabecular myocardium derives from embryonic careg $^{+}$CMs. $N=9$.

less differentiated contractile structures. Second, careg-positive CMs displayed a slightly bent shape with their ends facing the ventricular chamber, suggesting the formation of attachments with trabecular CMs (Fig. 8b). To determine whether the careg-positive cell layer indeed interconnects with the trabecular myocardium, we performed antibody staining against $\mathrm{N}$-cadherin. We found an enriched punctate pattern of $\mathrm{N}$-cadherin immunostaining in careg-positive cells, facing the trabecular but not the cortical myocardium (Fig. 8d), suggesting an uneven amount of adhesion on both surfaces of these cells. Thus, careg-expressing CMs contain structural features of junctional/transistional CMs, forming the circumference of the trabecular myocardium.

Electron microscopy analysis has identified the presence of a fibroblast network in the junctional region ${ }^{32}$. To determine the spatial distribution of the primordial layer in this zone, we generated double transgenic fish careg:dmKO2;wt1a-6.8kb:GFP, the latter of which demarcates the subcortical non-myocytes ${ }^{43,47}$. We found that most wt1a-6.8kb:GFP-positive cells were arranged between the cortical myocardium and careg-labelled CMs (Fig. 8e). Thus, a fibroblast layer seems to 'glue' together the 


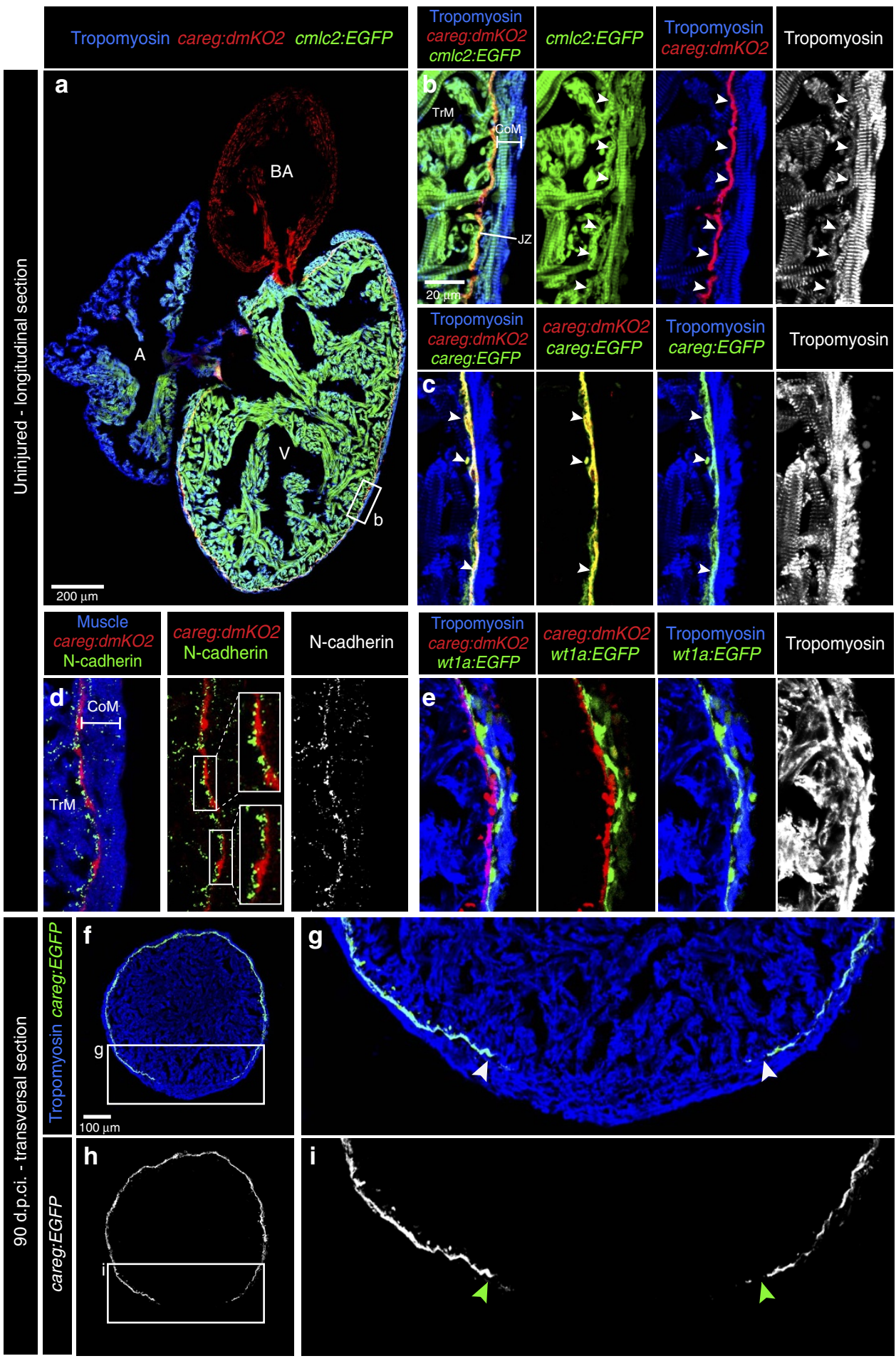

Figure 8 | Monitoring the primordial CM layer between the compact and trabecular myocardium. (a) Longitudinal sections of careg:dmKO2; $c m / c 2: E G F P$ transgenic adult hearts labelled with antibodies against $\mathrm{KO} 2$ (red) and Tropomyosin (blue, white). $\mathrm{V}$, ventricle; A, atrium; BA, bulbus arteriosus.

(b) careg: $\mathrm{dmKO2}$ expression is maintained during adulthood in a single layer of thin subcortical CMs (arrowheads) in the junctional zone (JZ) between the compact/cortical (CoM) and the trabecular myocardium ( $\operatorname{TrM}) . N=4$. (c) A higher magnification of the adult ventricle wall of double transgenic fish careg:dmKO2; careg:EGFP displays an overlapping expression of both markers in subcortical myocytes (arrowheads). $N=4$. (d) A magnified adult ventricle wall of careg:dmKO2 transgenic fish displays an abundant expression of $\mathrm{N}$-cadherin (green, white) on the surface of the junctional $\mathrm{CMs}$ on the side facing the trabecular myocardium. $N=4$. (e) A fragment of the adult ventricle wall of careg:dmKO2;wt7a-6.8kb:GFP transgenic fish displays the separation of compact myocardium and subcortical careg:dmKO2 ${ }^{+}$layer by wt7a-6.8kb:GFP-labelled fibroblasts of the junctional zone. $N=4$. (f-i) At 90 d.p.ci., transversal heart sections of careg:EGFP hearts display a gap in the primordial layer within the regenerated myocardium. The extent of the gap is indicated with arrowheads. $N=4$. 
cortical and primordial CMs. This suggests that the primordial layer is partially separated from the cortical layer by connective tissue.

Incomplete regeneration of the primordial cardiac layer. After injury, heart regeneration is completed within 30 to 60 days. Closer examination of careg:EGFP or careg: $d m K O 2$ transgenic fish revealed that the primordial/junctional CM layer was interrupted in the new myocardium, indicating its incomplete restoration, even at 90 d.p.ci. (Fig. 8f-i; Supplementary Fig. 3i,j). Thus, the regenerative competence of this CM layer is unexpectedly lower than that of other CMs.

To directly test the regenerative contribution of primordial/ junctional careg-positive CMs, we performed lineage tracing of these cells. To this aim, we crossed careg:CreERT2 with the cmlc2:loxP-AmCyan-STOP-loxP-ZsYellow lineage-tracing line, referred to as cmlc2:Switch, which labels cardiac cells in yellow after Cre-lox mediated recombination (Fig. 9a). In the absence of 4-OHT, no ZsYellow was detected in the ventricle sections of careg:CreERT2;cmlc2:Switch double transgenic fish (Supplementary Fig. 10a-c). To label the primordial CM layer, we pulsed adult transgenic fish careg:CreERT2;cmlc2:Switch with 4-OHT for 2 days, and we collected the hearts after 7 days (Supplementary Fig. 10d,e). We found that $\sim 30 \%(29.6 \pm 2.5$; $N=6)$ of the entire compact myocardium was demarcated by ZsYellow expression.

To determine whether primordial cells provide a source for the peri-injured trabecular myocardium, we performed cryoinjury 8 days after labelling of $\mathrm{CMs}$, and analysed the hearts at 10 d.p.ci. (Fig. 9a,b). Although the primordial layer of the remaining myocardium displayed ZsYellow expression, no fluorescent labelling was detected in the peri-injury zone of the trabecular myocardium (Fig. 9c,d). These fate-mapping results indicate that careg-positive primordial CMs, which have been labelled in the intact heart, do not markedly contribute to the dedifferentiation zone in the regenerating heart.

To examine the contribution of the primordial/junctional CMs in the regenerated ventricles, we used the same experimental design (Fig. 9a,b), and analysed the hearts at 30 d.p.ci. We found the main portion of the new myocardium was not markedly labelled by zsYellow expression, suggesting a relatively low regenerative contribution of primordial/junctional CMs. Moreover, the outer wall of the new myocardium contained gaps of zsYellow-labelled cells (Fig. 9e,f). This lineage-tracing analysis indicates that the primordial/junctional cardiac layer is incompletely restored after regeneration, which is consistent with careg:EGFP or careg:dmKO2 reporter results.

Regenerative contribution of peri-injury CMs and MES. To examine whether careg is induced de novo in the dedifferentiated trabecular CMs and to trace the destiny of careg-expressing cells during regeneration, we performed cell labelling after cryoinjury and monitored the distribution of the ZsYellow-positive CMs during new myocardium formation. To this aim, we performed cryoinjury of careg:CreERT2;cmlc2:Switch double transgenic fish, and gave animals a 4-OHT pulse between 5 and 7 d.p.ci. (Fig. 9g). Analysis of ventricle sections at 15 d.p.ci. revealed $55.1 \% \pm 2.6$ $(N=5)$ of ZsYellow-positive CMs in the peri-injury zone (Fig. 9h,i). At 30 d.p.ci., the injured tissue was replaced with a new myocardium that was abundantly labelled by ZsYellow-positive CMs within the trabecular and cortical regions (Fig. 9j,k). We concluded that the injury-abutting CMs from the trabecular and cortical myocardium were activated and provided a cellular source for the regenerated cardiac muscle. Thus, the lineagetracing analysis revealed that non-primordial/junctional CMs have a high regenerative capacity and contribute to new myocardium formation.

To examine whether careg is induced de novo in the activated stump mesenchyme of the fin, we performed cell labelling before and after amputation using careg:CreERT2; ubi:Switch transgenic fish. First, we gave animals a 1-day pulse of $4-\mathrm{OHT}$ at 10 days before amputation, and monitored mCherry expression during regeneration (Supplementary Fig. 10f). Live-imaging and immunofluorescence analysis of fin sections did not reveal labelling, except for a few scattered cells (Supplementary Fig. 10g-p). However, when the animals were exposed to 4-OHT during the first day following amputation, the regenerative outgrowth was demarcated by mCherry, as assessed by live-imaging of the fins at different time points, and immunofluorescence analysis of longitudinal sections at 4 d.p.a. (Fig. 9l-u). We concluded that careg is induced in the pre-existing MES of the stump, which gives rise to the new MES of the regenerate. Taken together, the regeneration-competent functional cells of the zebrafish heart and fin activate the careg element, indicating a common regulatory mechanism of organ restoration that operates in distinct cell types, such as cardiac myocytes and fin fibroblasts.

\section{Discussion}

Organ regeneration involves a morphogenetic stimulation of the remaining tissue in the peri-injury zone. Our findings uncovered that the local regenerative response operates through common regulatory mechanisms in different body structures, such as cardiac and mesenchymal tissues. We identified a genetic element, called careg, which is transiently activated in the periinjured CMs and fin MES during regeneration (Fig. 10). The identification of this transgenic biosensor supports the notion of regeneration-specific enhancers, which have been recently shown to act in the endocardium and the distal blastema ${ }^{30}$. Here we showed that during organ regeneration, the expression of careg in CMs and fin MES is dependent on TGF $\beta /$ Activin- $\beta$ signalling. Previous studies from our laboratory have identified an enhanced expression of TGF $\beta /$ Activin- $\beta$ ligands in wounded tissues of the heart and the fin, as well as the requirement of this pathway in organ restoration ${ }^{36,42}$. Remarkably, the careg reporter was not induced in all TGF $\beta /$ Activin- $\beta$-activated tissues after injury. In the heart, it was absent in the fibrotic area of the injured ventricle, despite the abundant number of pSmad3-positive cells and the requirement of TGF $\beta /$ Activin- $\beta$ for the beneficial extracellular matrix deposition in this zone $e^{42,43}$. Thus, the responsiveness of the careg reporter to TGF- $\beta$ signalling occurs specifically in regeneration-contributing cells and not in all regeneration-assisting cells of the injured heart. This finding indicates that careg should not be viewed as a universal responsive element of the TGF $\beta$ /Activin- $\beta$ activity in zebrafish. We propose that the careg element represents a common biosensor that is triggered by TGF $\beta /$ Activin- $\beta$ specifically in regenerating cells, such as CMs and MES, after injury of distinct zebrafish organs.

The careg element includes a $3.18 \mathrm{~kb}$ sequence immediately upstream of the ctgfa transcriptional start site. The bioinformatics analysis of this fragment indeed revealed a potential binding site for Smad3, but also for multiple other transcription factors. Thus, consistently with a previous study ${ }^{37}$, the careg element may represent a combination of various enhancers, which require further biochemical and genetic examination. Although careg comprises the upstream sequence of the ctgfa gene, the latter is not upregulated in regenerating CMs and MES. This differential expression pattern suggests that the full endogenous genomic sequence might include potential regeneration-suppressor binding sites, which prevent ctgfa expression in activated 
a
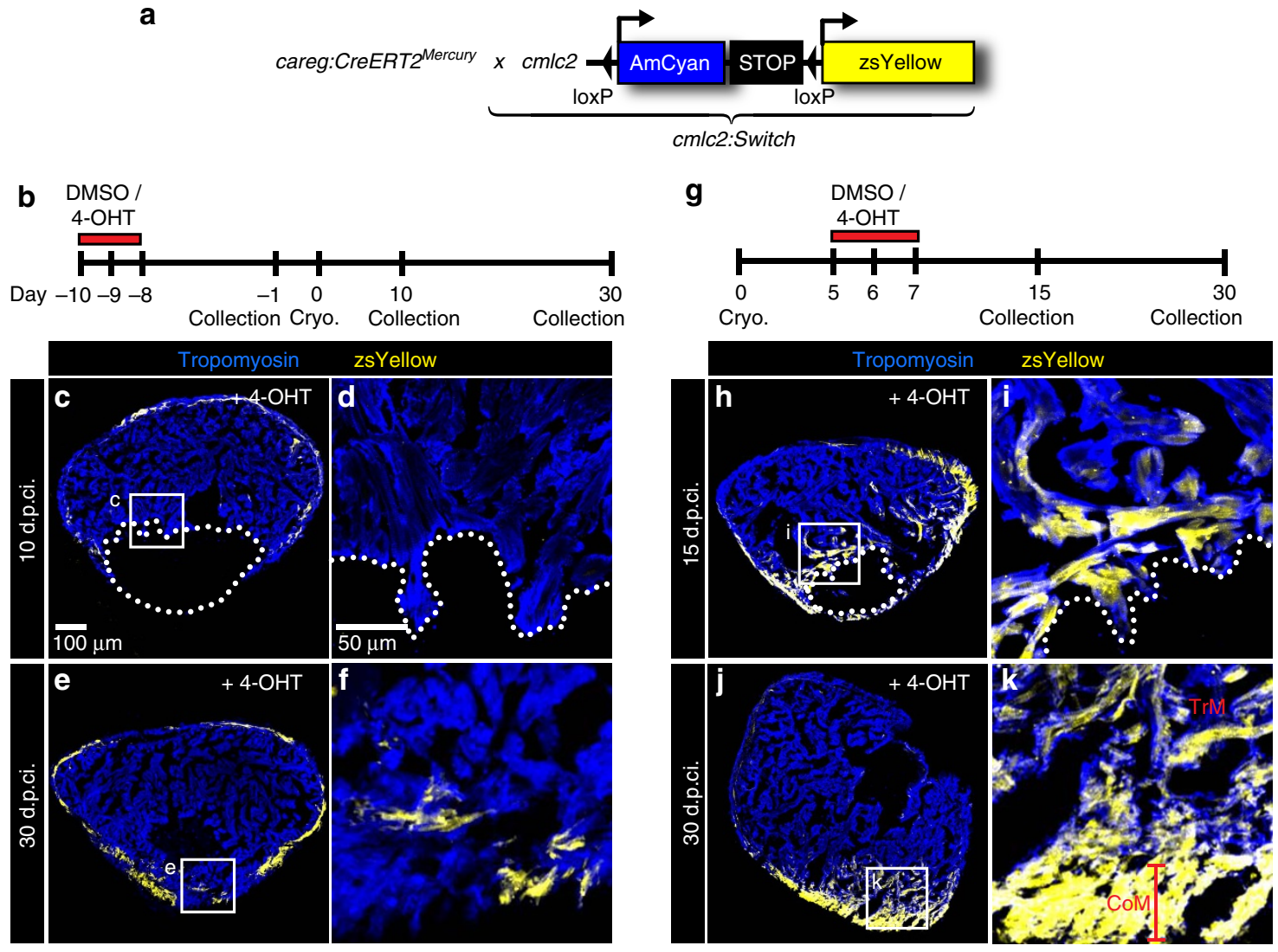

I
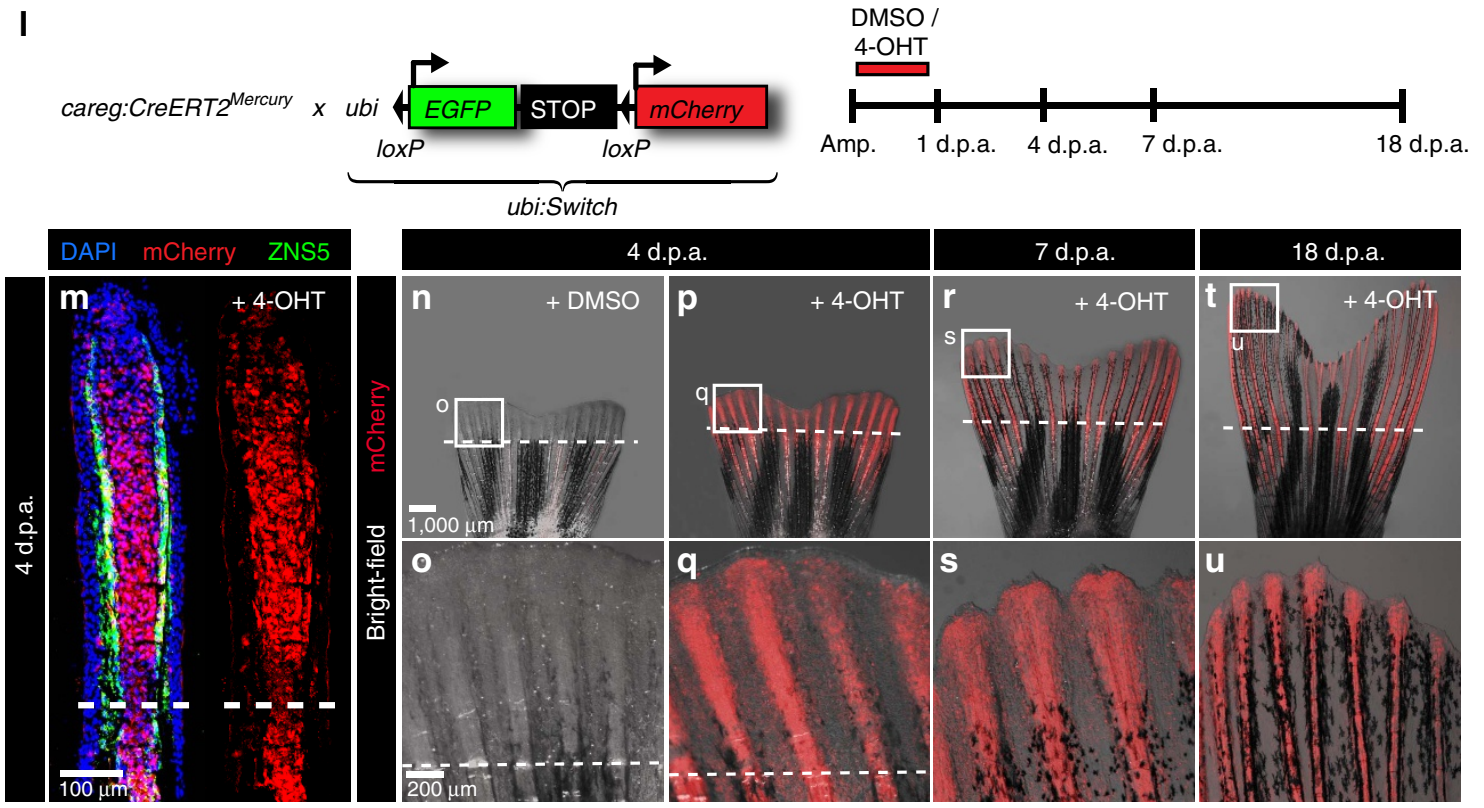

Figure 9 | careg-expressing cells contribute to the regenerating myocardium and fin mesenchyme. (a,b) Schematic representation of the transgenic strains and the experimental design for lineage tracing of careg-expressing primordial CMs during ventricle regeneration. (c-f) Representative sections of hearts treated with 4-OHT before cryoinjury to label the primordial layer, stained with antibody against Tropomyosin (blue). (c,d) At 10 d.p.ci., no zsYellow is detected in the trabecular myocardium at the injury site, indicating that primordial CMs do not contribute to the regeneration zone along the postcryolesioned tissue. (e,f) At 30 d.p.ci., the zsYellow-positive primordial layer regenerates incompletely. (g) Experimental design for lineage tracing of caregexpressing dedifferentiated CMs during ventricle regeneration. (h-k) Representative sections of hearts treated with 4-OHT after cryoinjury hearts immunostained against Tropomyosin (blue) (h,i) At 15 d.p.ci., zsYellow is expressed in the trabecular myocardium at the injury site. (j,k). At 30 d.p.ci., zsYellow-positive CMs are detected in the trabecular (TrM) and cortical myocardium (CoM) of the regenerated muscle. $N \geq 8$. (I) Transgenic strains and the design of lineage tracing experiments during fin regeneration. (m) Longitudinal section of lineage-labelled fins at 3 d.p.a., stained for osteoblasts using the Zns5 antibody (green) and for nuclei with DAPI (blue). mCherry is detected in the entire mesenchyme of the regenerative outgrowth and below the amputation plane. $N=6$. $(\mathbf{n}-\mathbf{u})$ Live-imaging of the same lineage-labelled fin at different time points of regeneration. The regenerative outgrowth and the stump tissue are labelled with mCherry. $N=6$. 
a
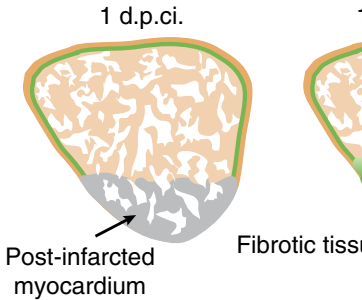

14 d.p.ci.

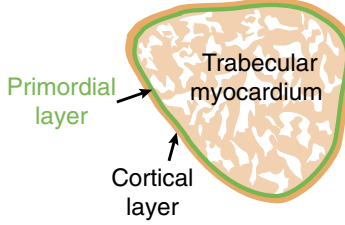

myocardium
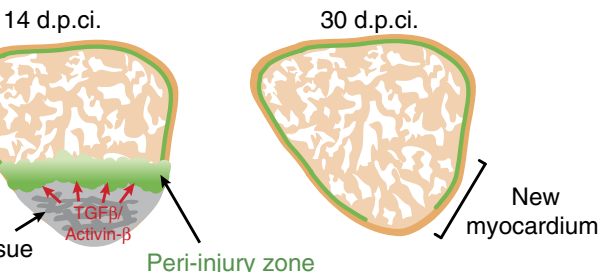

Peri-injury zone of the myocardium

b
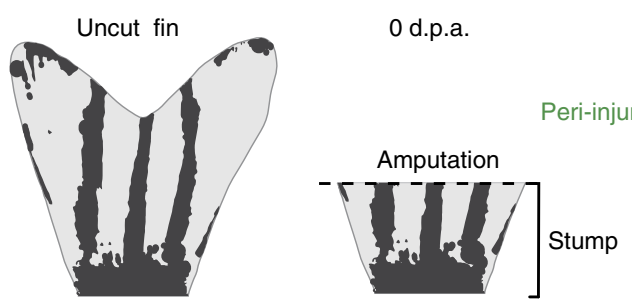

3 d.p.a.

Regenerative outgrowth

Careg-positive cells
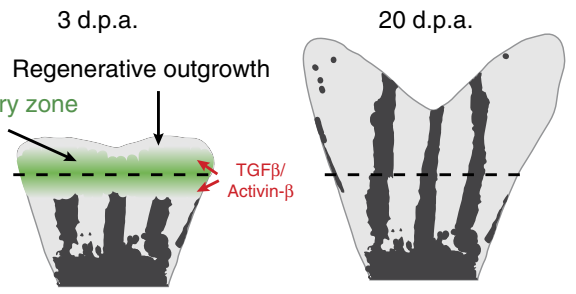

Figure 10 | CMs and stump MES activate a common regulatory element during regeneration. (a,b) A schematic representation of ventricle sections and whole fins at different time points during regeneration. The peri-injury zone of the ventricle and the stump of the fin activate a transient expression of the careg reporter in a TGF $\beta$ /Activin- $\beta$-dependent manner. In the heart, the careg reporter also demarcates the primordial layer, which fails to be completely restored in the new myocardium.

tissues. Further dissection of the adjacent genomic sequences beyond the careg element is required to identify potential negative regulatory elements. Indeed, a recent study on spinal cord regeneration established a ctgfa:EGFP transgenic fish line with a $5.5 \mathrm{~kb}$ sequence upstream of the ctgfa translational initiation $\operatorname{codon}^{48}$. It will be interesting to compare the expression of the careg and ctgfa reporters in the context of heart and fin regeneration.

Remarkably, the careg reporter is also constitutively expressed in the tissue-junctional layers, such as the primordial cardiac layer spanning the cortical and trabecular compartments, and the interface cells between bone matrix and MES in the fin. In the adult uninjured heart, our findings provide evidence that the primordial CM layer acts as a junctional/transitional layer, based on the architecture of the single-cell sheet, a flattened U-shaped cell morphology, an enrichment of $\mathrm{N}$-cadherins facing the trabecular fascicles and the presence of overlying connective tissue. These interconnecting $\mathrm{CMs}$ are probably exposed to certain stimuli that trigger the activation of the same regulatory elements as during regeneration, although in a TGF $\beta$ /Activin $-\beta$ independent manner. These stimuli remain unknown but they might be of biomechanical nature. Indeed, the junctional CM layer is thought to anchor the trabeculae with the luminal cavities in order to prevent compaction of the muscle ${ }^{49,50}$. In the locomotory appendage, osteoblasts connecting bones with MES may also cope with particular inter-tissue tension during swimming. Thus, the careg reporter is expressed in certain integrative cellular layers, which might be exposed to biophysical challenge during their normal function. Molecular mechanisms of such induction require further investigation.

The zebrafish ventricle comprises heterogeneous types of $\mathrm{CMs}^{32,33}$. Cortical CMs display a high regenerative potential, as previously shown using the gata4-reporter ${ }^{132}$. Our findings yielded several discoveries related to the regenerative competence of primordial and trabecular CMs. Lineage-tracing analysis in the embryo revealed that the primordial CM layer gives rise to the growing myocardium during development. In the adult heart, however, the primordial layer was not completely restored after cryoinjury, indicating its low ability to regenerate after traumatic injury. This limitation might be caused by the specialization of the primordial CM layer as the junctional layer of the heart wall.
By contrast, both the inner trabecular CMs, which build the main part of the ventricular chamber, and the cortical CMs, which surround the ventricular wall, all of them efficiently contribute to the formation of the new myocardium. It will be interesting to analyse gata4/careg double transgenic fish, to more accurately specify the relative contribution of cortical and trabecular CMs in the resection and cryoinjury models.

Our study of careg during fin regeneration allowed lineage tracing of the injury-activated MES. We show that MES of the regenerate arise from pre-existing local cells. This finding supports the concept that the newly formed tissue originates from the local stump and not from recruited remote cell populations ${ }^{8,29}$. The regenerative inadequacy in mammals is thought to result from the evolutionary loss of the intrinsic cellular plasticity that enables the activation of morphogenetic programs after injury. The identification of a common regulatory element in zebrafish heart and fin might provide a valuable resource for transgenic modulation of the regenerative response in other models.

\section{Methods}

Zebrafish lines and animal use. Wild-type $\mathrm{AB}$ (Oregon) and transgenic female and male adult zebrafish aged 6 to 12 months were used in this study. Genetically modified lines were: $\operatorname{dob}{ }^{45}, \operatorname{Tg}(\operatorname{ctgfa}: E G F P)^{38}$, which we renamed Tg(careg:EGFP), Tg(cmlc2:DsRed2-Nuc) ${ }^{51}$, Tg(cmlc2:EGFP $)^{52}, \mathrm{Tg}(\mathrm{cmlc2}$ : $\mathrm{loxP}$ AmCyan-loxP-ZsYellow), referred to as cmlc2:Switch ${ }^{53}$, ubi:loxP-EGFP-loxPmCherry (ubi:Switch $)^{54}$; $T g(w t 1 a-6.8 k b: G F P)^{55}$.

To generate careg lines, $3.18 \mathrm{~kb}$ upstream regulatory sequence of $\operatorname{ctgfa} \mathrm{a}^{37,38}$ was PCR-amplified from the plasmid pEGFP-1-ctgfa, which was kindly provided by Antonio Jacinto (Universidade Nova de Lisboa), with primers (F) $5^{\prime}$-CATGAGGAATGTTCCACTG- $3^{\prime}$ and (R) $5^{\prime}$-GAGAAGCAACAGTCACTC GAC- $3^{\prime}$. To establish careg:dmKO2 Kiwi, the PCR fragment was cloned into pDestTol2pA2 upstream of dmKO2 (ref. 39), kindly provided by Brian Link (Medical College of Wisconsin). To create careg:CreERT2 ${ }^{\text {Mercury }}$, the same PCR fragment of the ctgfa upstream regulatory sequence was cloned into p5E-MCS (\#473, zcre.org). The pDest_crya:Venus_careg:CreERT2 construct was generated using multisite Gateway assembly of p5E-ctgfa, pMECreERT2 (\#396, zcre.org), p3E_SV40polyA (\#302, zcre.org) and pDestTol2crya:Venus (\#480, zcre.org). Each plasmid was co-injected with the pCS2FA-transposase mRNA into one-cell-stage wild-type embryos.

All assays were performed using different animals that were randomly assigned to experimental groups. The exact sample size $(N)$ was described for each experiment in the figure legends, and was chosen to ensure the reproducibility of the results. During invasive procedures and imaging, fish were anaesthetized with buffered solution of $0.6 \mathrm{mM}$ tricaine (MS-222 ethyl-m-aminobenzoate, Sigma- 
Aldrich) in system water. Immediately after the procedures, zebrafish were transferred into a tank with system water, and were continuously monitored until fish restarted swimming. Fish normally resumed breathing within $30 \mathrm{~s}$ after transfer into water. In cases when the breathing was not observed after this time, we stimulated the animals by squirting water into their gills with a plastic pipette until they started spontaneous breathing. During regeneration, fish were maintained at $26.5^{\circ} \mathrm{C}$. Animal procedures were approved by the cantonal veterinary office of Fribourg, Switzerland.

Ventricular cryoinjury and resection. Myocardial cryoinjuries were performed according to the video protocol ${ }^{56}$. Briefly, anaesthetized fish were placed ventral side up in a damp sponge under the stereomicroscope. To access the heart, a small incision (approximately 1-2 mm) was made through the chest with iridectomy scissors (Roboz Surgical Instrument Co.), a procedure described as thoracotomy ${ }^{57}$. The beating heart was well visible, and no extensive bleeding occurred during the thoracotomy. Then, the ventricular wall was directly frozen by applying for 23-25 s a stainless steel cryoprobe (custom-made) pre-cooled in liquid nitrogen. The tip of the cryoprobe was $6 \mathrm{~mm}$ long with a diameter of $0.8 \mathrm{~mm}$, the handle of the cryoprobe was $4 \mathrm{~cm}$ long with a diameter of $8 \mathrm{~mm}$ and is covered with a plastic surface. To stop the freezing of the heart, system water at room temperature was dropped on the tip of the cryoprobe, and fish were immediately returned into a tank with water. It was not necessary to suture the incision of the chest, as the thoracic wound healed spontaneously within a week ${ }^{57,58}$.

Ventricular apex resection was performed according to the established protocol $^{40}$. Briefly, after thoracotomy, the apex of the ventricle was removed using curved iridectomy scissors.

To collect the heart for fixation, fish were killed in tricaine solution. A large $(\sim 4 \mathrm{~mm})$ incision was made above the heart through the branchial cartilage and the heart was pulled from the body cavity as shown in the video protocol ${ }^{56}$.

Fin cryoinjury and amputation. The fin cryoinjury was performed with a steel cryotome blade, $(22 \mathrm{~cm}, 260 \mathrm{~g}$, Product 14021660077 , Leica). The cryotome blade was immerged for $90 \mathrm{~s}$ in liquid nitrogen, removed and held for $5 \mathrm{~s}$ in the air to avoid the dispersion of liquid nitrogen droplets. The pre-cooled blade was placed for $15 \mathrm{~s}$ on the fin surface perpendicularly to the proximo-distal axis of the appendage at an equidistant position between the fin base and the central cleft ${ }^{41}$.

Fin amputations were performed using a razor blade. Adult caudal fins were amputated proximally to the first bone bifurcation point. Larval tails were cut at the posterior end of the notochord. Wound healing was spontaneous without significant bleeding. Live images of regenerating fins were taken with a Leica AF M205 FA stereomicroscope.

Drug treatments. The TGF $\beta$ type I receptor inhibitor SB431542 (Tocris) was dissolved in DMSO at a stock concentration of $20 \mathrm{mM}$ and used at a final concentration of $20 \mu \mathrm{M}$. Control animals were kept in water with $0.1 \%$ DMSO. For lineage tracing, fish were incubated in $5 \mu \mathrm{M}$ 4-hydroxytamoxifen (4-OHT, Tocris), which was made from a $10 \mathrm{mM}$ stock solution dissolved in DMSO, for 24 or $48 \mathrm{~h}$ at the time points indicated. For BrdU incorporation, fish were incubated for $48 \mathrm{~h}$ in water containing $50 \mu \mathrm{g} / \mathrm{ml} \mathrm{BrdU}$ (Sigma-Aldrich). Zebrafish were treated with drugs at a density of 3 fish per $100 \mathrm{ml}$ of water, and then returned to circulating system water.

Immunofluorescence analysis. Embryos and fins were fixed in $4 \%$ paraformaldehyde (PFA), adult heart in 2\% PFA, except the samples for $\mathrm{N}$-cadherin immunostaining, which were fixed in Dent's fixative ( $80 \%$ methanol, $20 \%$ dimethylsulfoxide). The fixation was performed overnight at $4{ }^{\circ} \mathrm{C}$, followed by washing in PBS ( $3 \times 10 \mathrm{~min}$ each). Embryos and heart specimens were equilibrated in $30 \%$ sucrose at $4{ }^{\circ} \mathrm{C}$. Fin specimens were first embedded in $1.5 \%$ agarose $/ 5 \%$ sucrose blocks, and then equilibrated in $30 \%$ sucrose. Subsequently, all specimens were embedded in tissue freezing media (Tissue-Tek O.C.T.; Sakura) and cryosectioned at a thickness of $12-16 \mu \mathrm{m}$. Sections were collected on Superfrost Plus slides (Fisher) and allowed to air dry for $\sim 1 \mathrm{~h}$ at room temperature (RT). The material was stored in tight boxes at $-20^{\circ} \mathrm{C}$.

Before use, slides were brought to room temperature for $10 \mathrm{~min}$, the area with sections was encircled with the ImmEdge Pen (Vector Laboratories) to keep liquid on the slides, and left for another $10 \mathrm{~min}$ at RT to dry. Then, the slides were transferred to coplin jars containing $0.3 \%$ Triton-X in PBS (PBST) for $10 \mathrm{~min}$ a RT. For BrdU and DsRed immunostainings, sections were incubated in $2 \mathrm{~N} \mathrm{HCl}$ in PBST during $35 \mathrm{~min}$ and washed $3 \times 10 \mathrm{~min}$ before the subsequent procedure.

The slides were transferred to a humid chamber. Blocking solution ( $5 \%$ goat serum in PBST) was applied on the sections for $1 \mathrm{~h}$ at RT. Subsequently, sections were covered with approx. $200 \mu \mathrm{l}$ of primary antibody diluted in blocking solution and incubated overnight at $4{ }^{\circ} \mathrm{C}$ in the humid chamber. They were washed in PBST in coplin jars for $1 \mathrm{~h}$ at RT and again transferred to the humid chamber for incubation with secondary antibodies diluted in blocking solution. The slides were washed in PBST for $1 \mathrm{~h}$ at RT and mounted in $90 \%$ glycerol in $20 \mathrm{mM}$ Tris pH 8 with $0.5 \% \mathrm{~N}$-propyl gallate.

The following primary antibodies were used: chicken anti-GFP at 1:2,000 (GFP1010, Aves Labs), rabbit anti-DsRed at 1:200 (632496, Clonetech), rabbit anti-KO2
1:200 (PM051M, Amalgaam) and mouse anti-KO2 1:500 (M168-3, Amalgaam), rabbit anti-mCherry 1:500 (GTX128508, GeneTex), mouse anti-embCMHC (N2.261) at 1:50 (developed by H.M. Blau, obtained from Developmental Studies Hybridoma Bank), mouse anti-tropomyosin at 1:100 (developed by J. Jung-Chin Lin and obtained from Developmental Studies Hybridoma Bank, CH1), rabbit antip-Smad3 at 1:400 (ab52903, Abcam), rat anti-BrdU at 1:100 (ab6326, Abcam), mouse anti-N-cadherin at 1:200 (610920, BD Biosciences), rabbit anti-Mef2c 1:50 (ab79436, Abcam), rabbit anti-Tenascin C 1:500 (T2550-23, US Biological); mouse Zns5 1:500 (ZDB-ATB-081002-37, ZFIN). The secondary antibodies (at 1:500) were Alexa conjugated (Jackson ImmunoResearch Laboratories). Phalloidin-Atto565 (94072, Sigma) and Atto-647 N (65906, Sigma) were used at 1:500. DAPI (Sigma) was applied to label nuclei.

In situ hybridization. The following forward (F) and reverse (R) primers $\left(5^{\prime}\right.$ to $\left.3^{\prime}\right)$ were used to synthesize the template for the probes: ctgfa (NM_001015041) F: $5^{\prime}$-TGTGGCTCAAGAGTGCAGTG- $3^{\prime}$ and R: $5^{\prime}$-CATGTCGCCAACCATCTTC-3', egfp (pEGFP-1); F: 5' -ACGTAAACGGCCACAAGTTC- $3^{\prime}$ and R: $5^{\prime}$-CTGGGTGCTCAGGTAGTGG-3', $m s x B$ (NM_131260) F: 5'-GAGAATGGGACATGGTCAGG-3 $3^{\prime}$ and R: 5'-GCGGTTCCTCAGGATAATAAC- $3^{\prime}$. To synthesize the anti-sense probes, a sequence of T3 RNA polymerase promoter ( $5^{\prime}$-ATTAACCCTCACTAAAGGGAGA-3') was added to the $5^{\prime}$ end of the reverse primers. The digoxigenin-RNA labelling mix (Roche) was used to generate the probe, which after purification was dissolved in hybridization solution and stored at $-20^{\circ} \mathrm{C}$.

In situ hybridization was performed on heart and fin cryosections. Organs were fixed overnight at $4{ }^{\circ} \mathrm{C}$ in $4 \%$ PFA, then washed twice in PBS, once in PBS/ methanol and in methanol for $10 \mathrm{~min}$ each, and finally transferred to methanol. Fixed material was stored at $-20^{\circ} \mathrm{C}$ until processed for cryosectioning, which was performed as for immunofluorescence. Before use, slides were brought to room temperature for $10 \mathrm{~min}$, and incubated in hybridization solution $(50 \%$ formamide, $5 \times$ SSC, $1 \times$ Denhardt's solution, $10 \%$ dextran sulphate, $0.1 \mathrm{mg} \mathrm{ml}^{-1}$ yeast tRNA; all from Sigma-Aldrich) for $1 \mathrm{~h}$ at RT and for $1 \mathrm{~h}$ at $60^{\circ} \mathrm{C}$. Then, they were placed into tight boxes with wet paper towels, and $200 \mu \mathrm{l}$ of probe diluted in hybridization solution (1:200) was applied on each slide that was then covered with a plastic coverslip (ApopTag, Merck Millipore) and placed in an incubator at $60{ }^{\circ} \mathrm{C}$ overnight. On the next day, slides were transferred to a coplin jar and soaked for 5 min with prewarmed $5 \times$ SSC (saline-sodium citrate) to allow the removal of cover slips, and washed twice with $5 \times$ SSC for $30 \mathrm{~min}$ and once with $0.2 \times$ SSC for $1 \mathrm{~h}$, each step at $60^{\circ} \mathrm{C}$. The final wash was done with $0.2 \times \mathrm{SSC}$ for $5 \mathrm{~min}$ at RT. Slides were allowed to dry for a few minutes at RT and sections was encircled with the ImmEdge Pen, and left for 15 min for drying. Slides were placed into humidified boxes, and $1 \times$ blocking reagent solution (Roche) in maleic acid buffer (100 mM Maleic Acid, $150 \mathrm{mM} \mathrm{NaCl}, 0.2 \%$ Tween-20, pH 7.5; all from SigmaAldrich) was applied on sections for $1 \mathrm{~h}$ at RT. Subsequently, $250 \mu \mathrm{l}$ of anti-digalkaline phosphatase (AP) antibody (Roche; diluted 1:4,000 in blocking reagent solution) was applied on sections for $2 \mathrm{~h}$ at RT. Slides were rinsed and washed twice for $30 \mathrm{~min}$ in maleic acid buffer in coplin jars. Then, slides were washed twice in AP-buffer (100 mM Tris HCL pH 9.5, $100 \mathrm{mM} \mathrm{NaCl}, 50 \mathrm{mM} \mathrm{MgCl}$, and $0.2 \%$ Tween-20) for $5 \mathrm{~min}$. To prepare the staining reagents, NBT and BCIP compounds (Roche) were diluted in AP-buffer according to manufacturer's instruction with addition of $10 \%$ Polyvinyl alcohol (Sigma-Aldrich). The staining reaction was performed in a humidified box at $37^{\circ} \mathrm{C}$. The reaction was monitored under the microscope, and was stopped

by washes in coplin jars with PBS for $15 \mathrm{~min}, 70 \%$ ethanol for $1 \mathrm{~h}$, and $15 \mathrm{~min}$ in PBS. The slides with fins were mounted in Aquatex mounting medium (Merck Millipore).

For heart sections, after completion of in situ hybridization, we performed fluorescent immunostaining against cardiac Tropomyosin, in order to visualize the intact part of the myocardium in regenerating ventricles ${ }^{59}$. After immunofluorescence staining, slides were mounted in $90 \%$ glycerol in $20 \mathrm{mM}$ Tris pH 8 with $0.5 \% \mathrm{~N}$-propyl gallate. Immunofluorescence and corresponding brightfield images were taken using a Leica SP5 confocal microscope and a Zeiss microscope, respectively, and were superimposed using Adobe Photoshop.

Image and statistical analysis. Fluorescent images of sections were taken with a Leica confocal microscope (TCS SP5) and the image J 1.49c software was used for subsequent measurements. Live images of fins were taken with a Leica stereomicroscope. For quantification of positive/labelled nuclei, we calculated the proportion of immunostained cells per total number of DAPI-stained nuclei or cardiac nuclear marker using ImageJ 1.46r software. For quantification of positive CMs, we calculated the proportion of immunostained area per either Tropomyosin- or F-actin-labelled tissue. Error bars correspond to standard error of the mean (s.e.m.). Significance of differences was calculated using unpaired Student's $t$-test. Statistical analyses were performed with the GraphPad Prism software. All results are expressed as the mean \pm s.e.m.

Sequence and bioinformatical analysis. An upstream genomic fragment of $\operatorname{ctg} f a$ was amplified from genomic DNA of wild-type AB zebrafish with the following primers: F: $5^{\prime}$-GAGCAGTGAGAGATGCATGG-3' and R: $5^{\prime}$-CCTCCTGATCGT 
GTTGAGTG- $3^{\prime}$. The PCR template was used for sequencing. The sequencing result was aligned to the careg sequence to verify the sequence similarity.

The LastZ sequence alignment programme ${ }^{60}$ from ENSEMBL Compara ${ }^{61}$ was used to build pairwise alignment of the ctgfa genomic region between zebrafish (Danio rerio, Ensembl version 87.10 ) and the following species: cavefish (Astyanax mexicanus), spotted gar (Lepisosteus oculatus), coelacanth (Latimeria chalumnae), tilapia (Oreochromis niloticus), platyfish (Xiphophorus maculatus), medaka (Oryzias latipes), fugu (Takifugu rubripes), stickleback (Gasterosteus aculeatus), tetraodon (Tetraodon nigroviridis), Xenopus (Xenopus tropicalis), chicken (Gallus gallus), mouse (Mus musculus), human (Homo sapiens).

Potential binding sites of transcription factors were predicted with the MatInsepctor software (MatInspector Release professional 8.3, December 2016, Genomatix Software Suite) ${ }^{62}$.

Data availability. The authors declare that all data supporting the findings of this study are available within the article and its Supplementary Information files, or from the corresponding author upon reasonable request.

\section{References}

1. Sehring, I. M., Jahn, C. \& Weidinger, G. Zebrafish fin and heart: what's special about regeneration? Curr. Opin. Genet. Dev. 40, 48-56 (2016).

2. Rubin, N., Harrison, M. R., Krainock, M., Kim, R. \& Lien, C. L. Recent advancements in understanding endogenous heart regeneration-insights from adult zebrafish and neonatal mice. Semin. Cell Dev. Biol. 58, 34-40 (2016).

3. Jaźwińska, A. \& Sallin, P. Regeneration versus scarring in vertebrate appendages and heart. J. Pathol. 238, 233-246 (2016).

4. Kikuchi, K. Dedifferentiation, transdifferentiation, and proliferation: mechanisms underlying cardiac muscle regeneration in zebrafish. Curr Pathobiol. Rep. 3, 81-88 (2015).

5. Foglia, M. J. \& Poss, K. D. Building and re-building the heart by cardiomyocyte proliferation. Development 143, 729-740 (2016).

6. Szibor, M., Poling, J., Warnecke, H., Kubin, T. \& Braun, T. Remodeling and dedifferentiation of adult cardiomyocytes during disease and regeneration. Cell Mol. Life Sci. 71, 1907-1916 (2014).

7. Pfefferli, C. \& Jaźwińska, A. The art of fin regeneration in zebrafish. Regeneration 2, 72-83 (2015).

8. Simon, A. \& Tanaka, E. M. Limb regeneration. Wiley Interdiscip. Rev. Dev. Biol. 2, 291-300 (2013)

9. Kumar, A. \& Brockes, J. P. Nerve dependence in tissue, organ, and appendage regeneration. Trends Neurosci. 35, 691-699 (2012).

10. Godwin, J. W. \& Rosenthal, N. Scar-free wound healing and regeneration in amphibians: immunological influences on regenerative success. Differentiation 87, 66-75 (2014).

11. Kragl, M. et al. Cells keep a memory of their tissue origin during axolotl limb regeneration. Nature 460, 60-65 (2009).

12. Jopling, C. et al. Zebrafish heart regeneration occurs by cardiomyocyte dedifferentiation and proliferation. Nature 464, 606-609 (2010).

13. Kikuchi, K. et al. Primary contribution to zebrafish heart regeneration by gata4 (+) cardiomyocytes. Nature 464, 601-605 (2010).

14. Singh, S. P., Holdway, J. E. \& Poss, K. D. Regeneration of amputated zebrafish fin rays from de novo osteoblasts. Dev. Cell 22, 879-886 (2012).

15. Sousa, S. et al. Differentiated skeletal cells contribute to blastema formation during zebrafish fin regeneration. Development 138, 3897-3905 (2011).

16. Sandoval-Guzman, T. et al. Fundamental differences in dedifferentiation and stem cell recruitment during skeletal muscle regeneration in two salamander species. Cell Stem Cell 14, 174-187 (2014).

17. Stewart, S. \& Stankunas, K. Limited dedifferentiation provides replacement tissue during zebrafish fin regeneration. Dev. Biol. 365, 339-349 (2012).

18. Knopf, F. et al. Bone regenerates via dedifferentiation of osteoblasts in the zebrafish fin. Dev. Cell 20, 713-724 (2011).

19. Tata, P. R. \& Rajagopal, J. Cellular plasticity: 1712 to the present day. Curr. Opin. Cell Biol. 43, 46-54 (2016).

20. Tu, S. \& Johnson, S. L. Fate restriction in the growing and regenerating zebrafish fin. Dev. Cell 20, 725-732 (2011).

21. Gupta, V. et al. An injury-responsive gata4 program shapes the zebrafish cardiac ventricle. Curr. Biol. 23, 1221-1227 (2013).

22. Nechiporuk, A. \& Keating, M. T. A proliferation gradient between proximal and msxb-expressing distal blastema directs zebrafish fin regeneration. Development 129, 2607-2617 (2002).

23. Wehner, D. et al. Wnt/beta-catenin signaling defines organizing centers that orchestrate growth and differentiation of the regenerating zebrafish caudal fin. Cell Rep. 6, 467-481 (2014).

24. Wehner, D. \& Weidinger, G. Signaling networks organizing regenerative growth of the zebrafish fin. Trends Genet. 31, 336-343 (2015).

25. Blum, N. \& Begemann, G. Retinoic acid signaling spatially restricts osteoblasts and controls ray-interray organization during zebrafish fin regeneration. Development 142, 2888-2893 (2015).
26. Stewart, S., Gomez, A. W., Armstrong, B. E., Henner, A. \& Stankunas, K. Sequential and opposing activities of Wnt and BMP coordinate zebrafish bone regeneration. Cell Rep. 6, 482-498 (2014).

27. Pfefferli, C., Muller, F., Jaźwińska, A. \& Wicky, C. Specific NuRD components are required for fin regeneration in zebrafish. BMC. Biol. 12, 30 (2014).

28. Xiao, C. et al. Chromatin-remodelling factor Brg1 regulates myocardial proliferation and regeneration in zebrafish. Nat. Commun. 7, 13787 (2016).

29. Tornini, V. A. et al. Live monitoring of blastemal cell contributions during appendage regeneration. Curr. Biol. 26, 2981-2991 (2016).

30. Kang, J. et al. Modulation of tissue repair by regeneration enhancer elements. Nature 532, 201-206 (2016).

31. Hu, N., Yost, H. J. \& Clark, E. B. Cardiac morphology and blood pressure in the adult zebrafish. Anat. Rec. 264, 1-12 (2001).

32. Lafontant, P. J. et al. Cardiac myocyte diversity and a fibroblast network in the junctional region of the zebrafish heart revealed by transmission and serial block-face scanning electron microscopy. PLoS ONE 8, e72388 (2013).

33. Icardo, J. in Ontogeny and Phylogeny of the Vertebrate Heart (eds Wang, T. \& Sedmera, D.) Ch. 2 35-53 (Springer, 2012).

34. Gupta, V. \& Poss, K. D. Clonally dominant cardiomyocytes direct heart morphogenesis. Nature 484, 479-484 (2012).

35. Sallin, P., de Preux Charles, A. S., Duruz, V., Pfefferli, C. \& Jaźwińska, A. A dual epimorphic and compensatory mode of heart regeneration in zebrafish. Dev. Biol. 399, 27-40 (2015).

36. Jaźwińska, A., Badakov, R. \& Keating, M. T. Activin-betaA signaling is required for zebrafish fin regeneration. Curr. Biol. 17, 1390-1395 (2007).

37. Chiou, M. J., Chao, T. T., Wu, J. L., Kuo, C. M. \& Chen, J. Y. The physiological role of CTGF/CCN2 in zebrafish notochond development and biological analysis of the proximal promoter region. Biochem. Biophys. Res. Commun. 349, 750-758 (2006).

38. Mateus, R. et al. Control of tissue growth by Yap relies on cell density and F-actin in zebrafish fin regeneration. Development 142, 2752-2763 (2015).

39. Collery, R. F. \& Link, B. A. Dynamic smad-mediated BMP signaling revealed through transgenic zebrafish. Dev. Dyn. 240, 712-722 (2011).

40. Poss, K. D., Wilson, L. G. \& Keating, M. T. Heart regeneration in zebrafish. Science 298, 2188-2190 (2002).

41. Chassot, B., Pury, D. \& Jaźwińska, A. Zebrafish fin regeneration after cryoinjury-induced tissue damage. Biol. Open 5, 819-828 (2016).

42. Chablais, F. \& Jaźwińska, A. The regenerative capacity of the zebrafish heart is dependent on TGFbeta signaling. Development 139, 1921-1930 (2012).

43. Marro, J., Pfefferli, C., de Preux Charles, A. S., Bise, T. \& Jaźwińska, A. Collagen XII contributes to epicardial and connective tissues in the zebrafish heart during ontogenesis and regeneration. PLoS ONE 11, e0165497 (2016).

44. Macias, M. J., Martin-Malpartida, P. \& Massague, J. Structural determinants of Smad function in TGF-beta signaling. Trends Biochem. Sci. 40, 296-308 (2015).

45. Whitehead, G. G., Makino, S., Lien, C. L. \& Keating, M. T. fgf20 is essential for initiating zebrafish fin regeneration. Science 310, 1957-1960 (2005).

46. Liu, J. et al. A dual role for ErbB2 signaling in cardiac trabeculation. Development 137, 3867-3875 (2010).

47. Peralta, M., Gonzalez-Rosa, J. M., Marques, I. J. \& Mercader, N. The Epicardium in the Embryonic and Adult Zebrafish. J. Dev. Biol. 2, 101-116 (2014).

48. Mokalled, M. H. et al. Injury-induced ctgfa directs glial bridging and spinal cord regeneration in zebrafish. Science 354, 630-634 (2016).

49. Burggren, W. W. et al. Comparative cardiovascular physiology: future trends, opportunities and challenges. Acta Physiol. 210, 257-276 (2014).

50. Pieperhoff, S., Bennett, W. \& Farrell, A. P. The intercellular organization of the two muscular systems in the adult salmonid heart, the compact and the spongy myocardium. J. Anat. 215, 536-547 (2009).

51. Rottbauer, W. et al. Reptin and pontin antagonistically regulate heart growth in zebrafish embryos. Cell 111, 661-672 (2002).

52. Burns, C. G. et al. High-throughput assay for small molecules that modulate zebrafish embryonic heart rate. Nat. Chem. Biol. 1, 263-264 (2005).

53. Zhou, Y. et al. Latent TGF-beta binding protein 3 identifies a second heart field in zebrafish. Nature 474, 645-648 (2011).

54. Mosimann, C. et al. Ubiquitous transgene expression and Cre-based recombination driven by the ubiquitin promoter in zebrafish. Development 138, 169-177 (2011).

55. Bollig, F. et al. A highly conserved retinoic acid responsive element controls wtla expression in the zebrafish pronephros. Development 136, 2883-2892 (2009).

56. Chablais, F. \& Jaźwińska, A. Induction of myocardial infarction in adult zebrafish using cryoinjury. J. Vis. Exp. 62, e3666 (2012).

57. de Preux Charles, A. S., Bise, T., Baier, F., Sallin, P. \& Jaźwińska, A. Preconditioning boosts regenerative programmes in the adult zebrafish heart. Open Biol. 6, 160101 (2016).

58. de Preux Charles, A. S., Bise, T., Baier, F., Marro, J. \& Jaźwińska, A. Distinct effects of inflammation on preconditioning and regeneration of the adult zebrafish heart. Open Biol. 6, 160102 (2016). 
59. Sallin, P. \& Jaźwińska, A. Acute stress is detrimental to heart regeneration in zebrafish. Open Biol. 6, 160012 (2016).

60. Harris, M. S. Improved pairwise alignment of genomic DNA. Ph.D. Thesis, The Pennsylvania State Univ. (2007).

61. Herrero, J. et al. Ensembl comparative genomics resources. Database 2016, bav096 (2016)

62. Cartharius, K. et al. MatInspector and beyond: promoter analysis based on transcription factor binding sites. Bioinformatics 21, 2933-2942 (2005).

\section{Acknowledgements}

We thank V. Zimmermann for excellent technical assistance and for fish care, A. Puoti, S. Käser-Pébernard and D. König for critical reading of the manuscript. We are grateful to Laurent Falquet, for help with the bioinformatics analysis; B. Link (Medical College of Wisconsin) for sending the dmKO2 plasmid; A. Jacinto (Universidade Nova de Lisboa) for sharing ctgfa:EGFP transgenic fish; C. Mosimann (University of Zürich) for providing ubi:Switch and cmlc2:Switch strains. This work was supported by the Swiss National Science Foundation, grant numbers: 310030_159995 and CRSII3_147675, and the Swiss Heart Foundation (Die Schweizerische Herzstiftung).

\section{Author contributions}

C.P. planned, performed experiments and analysed the data. A.J. conceived and supervised the study, designed experiments and wrote the manuscript with input from C.P.

\section{Additional information}

Supplementary Information accompanies this paper at http://www.nature.com/ naturecommunications

Competing interests: The authors declare no competing financial interests.

Reprints and permission information is available online at http://npg.nature.com/ reprintsandpermissions/

How to cite this article: Pfefferli, C. \& Jaźwińska, A. The careg element reveals a common regulation of regeneration in the zebrafish myocardium and fin. Nat. Commun. 8, 15151 doi: $10.1038 /$ ncomms15151 (2017).

Publisher's note: Springer Nature remains neutral with regard to jurisdictional claims in published maps and institutional affiliations.

(c) (i) This work is licensed under a Creative Commons Attribution 4.0 International License. The images or other third party material in this article are included in the article's Creative Commons license, unless indicated otherwise in the credit line; if the material is not included under the Creative Commons license, users will need to obtain permission from the license holder to reproduce the material. To view a copy of this license, visit http://creativecommons.org/licenses/by/4.0/

(C) The Author(s) 2017 